\title{
VARIATIONAL DISCRETIZATION OF A CONTROL-CONSTRAINED PARABOLIC BANG-BANG OPTIMAL CONTROL PROBLEM ${ }^{*}$
}

\author{
Nikolaus von Daniels and Michael Hinze \\ Schwerpunkt Optimierung und Approximation, Universitat Hamburg, Bundesstrase 55, \\ 20146 Hamburg, Germany \\ Email: nvdmath@gmx.net, michael.hinze@uni-hamburg.de
}

\begin{abstract}
We consider a control-constrained parabolic optimal control problem without Tikhonov term in the tracking functional. For the numerical treatment, we use variational discretization of its Tikhonov regularization: For the state and the adjoint equation, we apply Petrov-Galerkin schemes in time and usual conforming finite elements in space. We prove a-priori estimates for the error between the discretized regularized problem and the limit problem. Since these estimates are not robust if the regularization parameter tends to zero, we establish robust estimates, which — depending on the problem's regularity — enhance the previous ones. In the special case of bang-bang solutions, these estimates are further improved. A numerical example confirms our analytical findings.

Mathematics subject classification: 49J20, 35K20, 49J30, 49M05, 49M25, 49M29, 65M12, $65 \mathrm{M} 60$.

Key words: Optimal control, Heat equation, Control constraints, Finite elements, A-priori error estimates, Bang-bang controls.
\end{abstract}

\section{Introduction}

In this article we are interested in the numerical solution of the optimal control problem

$$
\min _{u \in U_{\text {ad }}} J_{0}(u) \quad \text { with } \quad J_{0}(u):=\frac{1}{2}\|T u-z\|_{H}^{2} .
$$

Here, $T$ is basically the (weak) solution operator of the heat equation, the set of admissible controls $U_{\text {ad }}$ is given by box constraints, and $z \in H$ is a given function to be tracked.

Often, the solutions of $\left(\mathbb{P}_{0}\right)$ possess a special structure: They take values only on the bounds of the admissible set $U_{\mathrm{ad}}$ and are therefore called bang-bang solutions.

Theoretical and numerical questions related to this control problem attracted much interest in recent years, see, e.g., [1-11]. The last four papers are concerned with $T$ being the solution operator of an ordinary differential equation, the former papers with $T$ being a solution operator of an elliptic PDE or $T$ being a continuous linear operator. In [12], a brief survey of the content of these and some other related papers is given at the end of the bibliography.

Problem $\left(\mathbb{P}_{0}\right)$ is in general ill-posed, meaning that a solution does not depend continuously on the datum $z$, see $\left[3\right.$, p. 1130]. The numerical treatment of a discretized version of $\left(\mathbb{P}_{0}\right)$ is also challenging, e.g., due to the absense of formula (2.10) in the case $\alpha=0$, which corresponds to problem $\left(\mathbb{P}_{0}\right)$.

\footnotetext{
* Received July 6, 2017 / Revised version received April 19, 2018 / Accepted May 7, 2018 /

Published online August 14, 2018 /
} 
Therefore we use Tikhonov regularization to overcome these difficulties. The regularized problem is given by

$$
\min _{u \in U_{\mathrm{ad}}} J_{\alpha}(u) \quad \text { with } \quad J_{\alpha}(u):=\frac{1}{2}\|T u-z\|_{H}^{2}+\frac{\alpha}{2}\|u\|_{U}^{2}
$$

where $\alpha>0$ denotes the regularization parameter. Note that for $\alpha=0$, problem $\left(\mathbb{P}_{\alpha}\right)$ reduces to problem $\left(\mathbb{P}_{0}\right)$.

For the numerical treatment of the regularized problem, we then use variational discretization introduced by Hinze in [13], see also [14, Chapter 3.2.5]. The state equation is treated with a Petrov-Galerkin scheme in time using a piecewise constant Ansatz for the state and piecewise linear, continuous test functions. This results in variants of the Crank-Nicolson scheme for the discretization of the state and the adjoint state, which were proposed recently in [15]. In space, usual conforming finite elements are taken. See [12] for the fully discrete case and [16] for an alternative discontinuous Galerkin approach.

The purpose of this paper is to prove a-priori bounds for the error between the discretized regularized problem and the limit problem, i.e. the continuous unregularized problem.

We first derive error estimates between the discretized regularized problem and its continuous counterpart. Together with Tikhonov error estimates recently obtained in [17], see also [12], one can establish estimates for the total error between the discretized regularized solution and the solution of the continous limit problem, i.e. $\alpha=0$. Here, second order convergence in space is not achievable and (without coupling) the estimates are not robust if $\alpha$ tends to zero. Using refined arguments, we overcome both drawbacks. In the special case of bang-bang controls, we further improve those estimates.

The obtained estimates suggest a coupling rule for the parameters $\alpha$ (regularization parameter), $k$, and $h$ (time and space discretization parameters, respectively) to obtain optimal convergence rates which we numerically observe.

The paper is organized as follows.

In the next section, we introduce the functional analytic description of the regularized problem. We recall several of its properties, such as existence of a unique solution for all $\alpha \geq 0$ (thus especially in the limit case $\alpha=0$ we are interested in), an explicit characterization of the solution structure, and the function space regularity of the solution. We then introduce the Tikhonov regularization and recall some error estimates under suitable assumptions. In the special case of bang-bang controls, we recall a smoothness-decay lemma which later helps to improve the error estimates for the discretized problem.

The third section is devoted to the discretization of the optimal control problem. At first, the discretization of the state and adjoint equation is introduced and several error estimates needed in the later analysis are recalled. Then, the analysis of variational discretization of the optimal control problem is conducted.

The last section discusses a numerical example where we observe the predicted orders of convergence.

\section{The Continuous Optimal Control Problem}

\subsection{Problem setting and basic properties}

Let $\Omega \subset \mathbb{R}^{d}, d \in\{2,3\}$, be a spatial domain which is assumed to be bounded and convex with a polygonal boundary $\partial \Omega$. Furthermore, a fixed time interval $I:=(0, T) \subset \mathbb{R}, 0<T<\infty$, 
a desired state $y_{d} \in L^{2}\left(I, L^{2}(\Omega)\right)$, a non-negative real constant $0 \leq \alpha \in \mathbb{R}$, and an initial value $y_{0} \in L^{2}(\Omega)$ are prescribed. With the Gelfand triple $H_{0}^{1}(\Omega) \hookrightarrow L^{2}(\Omega) \hookrightarrow H^{-1}(\Omega)$ we consider the following optimal control problem

$$
\begin{aligned}
& \min _{y \in Y, u \in U_{\text {ad }}} J(y, u) \quad \text { with } \quad J(y, u):=\frac{1}{2}\left\|y-y_{d}\right\|_{L^{2}\left(I, L^{2}(\Omega)\right)}^{2}+\frac{\alpha}{2}\|u\|_{U}^{2}, \\
& \text { s.t. } y=S\left(B u, y_{0}\right)
\end{aligned}
$$

where $U:=L^{2}\left(\Omega_{U}\right)$ is the control space, the (closed and convex) set of admissible controls is defined by

$$
U_{\mathrm{ad}}:=\left\{u \in U \mid a(x) \leq u(x) \leq b(x) \quad \forall^{\prime} x \in \Omega_{U}\right\}
$$

with fixed control bounds $a, b \in L^{\infty}\left(\Omega_{U}\right)$ fulfilling $a \leq b$ almost everywhere in $\Omega_{U}$,

$$
Y:=W(I):=\left\{v \in L^{2}\left(I, H_{0}^{1}(\Omega)\right) \mid v_{t} \in L^{2}\left(I, H^{-1}(\Omega)\right)\right\}
$$

is the state space, and the control operator $B$ as well as the control region $\Omega_{U}$ are defined below.

Note that we use the notation $v_{t}$ and $\partial_{t} v$ for weak time derivatives and $\forall^{\prime}$ for "for almost all".

The operator

$$
S: L^{2}\left(I, H^{-1}(\Omega)\right) \times L^{2}(\Omega) \rightarrow W(I), \quad(f, g) \mapsto y:=S(f, g),
$$

denotes the weak solution operator associated with the heat equation, i.e., the linear parabolic problem

$$
\begin{array}{ll}
\partial_{t} y-\Delta y=f & \text { in } I \times \Omega, \\
y=0 & \text { in } I \times \partial \Omega, \\
y(0)=g & \text { in } \Omega .
\end{array}
$$

The weak solution is defined as follows. For $(f, g) \in L^{2}\left(I, H^{-1}(\Omega)\right) \times L^{2}(\Omega)$ the function $y \in W(I)$ with $\langle\cdot, \cdot\rangle:=\langle\cdot, \cdot\rangle_{H^{-1}(\Omega) H_{0}^{1}(\Omega)}$ satisfies the two equations

$$
\begin{aligned}
& y(0)=g, \\
& \int_{0}^{T}\left\langle\partial_{t} y(t), v(t)\right\rangle+a(y(t), v(t)) d t=\int_{0}^{T}\langle f(t), v(t)\rangle d t, \quad \forall v \in L^{2}\left(I, H_{0}^{1}(\Omega)\right) .
\end{aligned}
$$

Note that by the embedding $W(I) \hookrightarrow C\left([0, T], L^{2}(\Omega)\right)$, see, e.g., [18, Theorem 5.9.3], the first relation is meaningful.

In the preceding equation, the bilinear form $a: H^{1}(\Omega) \times H^{1}(\Omega) \rightarrow \mathbb{R}$ is given by

$$
a(f, g):=\int_{\Omega} \nabla f(x) \nabla g(x) d x .
$$

We show below that (2.3) yields an operator $S$ in the sense of (2.2).

For the control region $\Omega_{U}$ and the control operator $B$ we consider two situations.

1. (Distributed controls) We set $\Omega_{U}:=I \times \Omega$, and define the control operator $B: U \rightarrow$ $L^{2}\left(I, H^{-1}(\Omega)\right)$ by $B:=\mathrm{Id}$, i.e., the identity mapping induced by the standard Sobolev embedding $L^{2}(\Omega) \hookrightarrow H^{-1}(\Omega)$. 
2. (Located controls) We set the control region $\Omega_{U}:=I$. With a fixed functional $g_{1} \in$ $H^{-1}(\Omega)$ the linear and continuous control operator $B$ is given by

$$
B: U=L^{2}(I) \rightarrow L^{2}\left(I, H^{-1}(\Omega)\right), \quad u \mapsto\left(t \mapsto u(t) g_{1}\right) .
$$

The case of $D$ fixed functionals $g_{i}$ with controls $u_{i}$ and a control operator $B: L^{2}\left(I, \mathbb{R}^{D}\right) \rightarrow$ $L^{2}\left(I, H^{-1}(\Omega)\right), u \mapsto\left(t \mapsto \sum_{i=1}^{D} u_{i}(t) g_{i}\right)$ is a possible generalization. To streamline the presentation we restrict ourselves to the case $D=1$ here and refer to [12] for the case $D>1$.

For later use we observe that the adjoint operator $B^{*}$ is given by

$$
B^{*}: L^{2}\left(I, H_{0}^{1}(\Omega)\right) \rightarrow U=L^{2}(I), \quad\left(B^{*} q\right)(t)=\left\langle g_{1}, q(t)\right\rangle_{H^{-1}(\Omega) H_{0}^{1}(\Omega)} .
$$

If furthermore $g_{1} \in L^{2}(\Omega)$ holds, we can consider $B$ as an operator $B: L^{2}(I) \rightarrow$ $L^{2}\left(I, L^{2}(\Omega)\right)$ and get the adjoint operator

$$
B^{*}: L^{2}\left(I, L^{2}(\Omega)\right) \rightarrow U=L^{2}(I), \quad\left(B^{*} q\right)(t)=\left(g_{1}, q(t)\right)_{L^{2}(\Omega)} .
$$

Note that the adjoint operator $B^{*}$ (and also the operator itself) is preserving time regularity, i.e., $B^{*}: H^{k}(I, X) \rightarrow H^{k}(I)$ for $k \geq 0$ where $X$ is a subspace of $L^{2}(\Omega)$ depending on the regularity of the $g_{1}$ (as noticed just before), e.g., $X=L^{2}(\Omega)$ or $X=H_{0}^{1}(\Omega)$.

\section{Lemma 2.1 (Properties of the solution operator $S$ ).}

1. For every $(f, g) \in L^{2}\left(I, H^{-1}(\Omega)\right) \times L^{2}(\Omega)$ a unique state $y \in W(I)$ satisfying (2.3) exists. Thus the operator $S$ from (2.2) exists. Furthermore the state fulfills

$$
\|y\|_{W(I)} \leq C\left(\|f\|_{L^{2}\left(I, H^{-1}(\Omega)\right)}+\|g\|_{L^{2}(\Omega)}\right) .
$$

2. Consider the bilinear form $A: W(I) \times W(I) \rightarrow \mathbb{R}$ given by

$$
A(y, v):=\int_{0}^{T}-\left\langle v_{t}, y\right\rangle+a(y, v) d t+\langle y(T), v(T)\rangle
$$

with $\langle\cdot, \cdot\rangle:=\langle\cdot, \cdot\rangle_{H^{-1}(\Omega) H_{0}^{1}(\Omega)}$. Then for $y \in W(I)$, Eq. (2.3) is equivalent to

$$
A(y, v)=\int_{0}^{T}\langle f, v\rangle d t+(g, v(0))_{L^{2}(\Omega)} \quad \forall v \in W(I) .
$$

Furthermore, $y$ is the only function in $W(I)$ fulfilling equation (2.7).

Proof. This can be derived using standard results, see [12, Lemma 1].

An advantage of the formulation (2.7) in comparison to (2.3) is the fact that the weak time derivative $y_{t}$ of $y$ is not part of the equation. Later in discretizations of this equation, it offers the possibility to consider states which do not possess a weak time derivative.

We can now establish the existence of a solution to problem $(\mathbb{P})$.

Lemma 2.2 (Unique solution of the o.c.p.). The optimal control problem $(\mathbb{P})$ admits for fixed $\alpha \geq 0$ a unique solution $\left(\bar{y}_{\alpha}, \bar{u}_{\alpha}\right) \in Y \times U$, which can be characterized by the first order necessary and sufficient optimality condition

$$
\bar{u}_{\alpha} \in U_{\mathrm{ad}}, \quad\left(\alpha \bar{u}_{\alpha}+B^{*} \bar{p}_{\alpha}, u-\bar{u}_{\alpha}\right)_{U} \geq 0 \quad \forall u \in U_{\mathrm{ad}},
$$


where $B^{*}$ denotes the adjoint operator of $B$, and the so-called optimal adjoint state $\bar{p}_{\alpha} \in W(I)$ is the unique weak solution defined and uniquely determined by the equation

$$
A\left(v, \bar{p}_{\alpha}\right)=\int_{0}^{T}\langle h, v\rangle_{H^{-1}(\Omega) H_{0}^{1}(\Omega)} d t \quad \forall v \in W(I)
$$

with right-hand side $h:=\bar{y}_{\alpha}-y_{d}$.

Proof. This follows from standard results, see, e.g., [12, Lemma 2].

As a consequence of the fact that $U_{\text {ad }}$ is a closed and convex set in a Hilbert space we have the following lemma.

Lemma 2.3. In the case $\alpha>0$ the variational inequality (2.8) is equivalent to

$$
\bar{u}_{\alpha}=P_{U_{\mathrm{ad}}}\left(-\frac{1}{\alpha} B^{*} \bar{p}_{\alpha}\right),
$$

where $P_{U_{\text {ad }}}: U \rightarrow U_{\text {ad }}$ is the orthogonal projection.

Proof. See [14, Corollary 1.2, p. 70] with $\gamma=\frac{1}{\alpha}$.

The orthogonal projection in (2.10) can be made explicit in our setting.

Lemma 2.4. Let us for $c_{1}, c_{2} \in \mathbb{R}$ with $c_{1} \leq c_{2}$ consider the projection of a real number $x \in \mathbb{R}$ into the interval $\left[c_{1}, c_{2}\right]$, i.e., $P_{\left[c_{1}, c_{2}\right]}(x):=\max \left\{c_{1}, \min \left\{x, c_{2}\right\}\right\}$.

There holds for $v \in U$ with $U_{\mathrm{ad}}$ defined in (2.1) the equation

$$
P_{U_{\mathrm{ad}}}(v)(x)=P_{[a(x), b(x)]}(v(x)) \quad \forall^{\prime} x \in \Omega_{U} .
$$

Proof. See [12, Lemma 4] for a proof of this standard result in our setting.

We now derive an explicit characterization of the optimal control.

Lemma 2.5. If $\alpha>0$, then for almost all $x \in \Omega_{U}$ there holds for the optimal control

$$
\bar{u}_{\alpha}(x)= \begin{cases}a(x) & \text { if } B^{*} \bar{p}_{\alpha}(x)+\alpha a(x)>0, \\ -\alpha^{-1} B^{*} \bar{p}_{\alpha}(x) & \text { if } B^{*} \bar{p}_{\alpha}(x)+\alpha \bar{u}_{\alpha}(x)=0, \\ b(x) & \text { if } B^{*} \bar{p}_{\alpha}(x)+\alpha b(x)<0 .\end{cases}
$$

Suppose $\alpha=0$ is given. Then the optimal control fulfills a.e.

$$
\bar{u}_{0}(x)= \begin{cases}a(x) & \text { if } B^{*} \bar{p}_{0}(x)>0, \\ b(x) & \text { if } B^{*} \bar{p}_{0}(x)<0 .\end{cases}
$$

Proof. We refer to [12, Lemma 5] for a proof of this standard result in our setting.

Remark 2.1. As a consequence of (2.12) we have: If $B^{*} \bar{p}_{0}$ vanishes only on a subset of $\Omega_{U}$ with Lebesgue measure zero, the optimal control $\bar{u}_{0}$ only takes values on the bounds $a, b$ of the admissible set $U_{\text {ad }}$. In this case $\bar{u}_{0}$ is called a bang-bang solution. 
Assuming more regularity on the data than stated above, we get regularity for the optimal state $\bar{y}_{\alpha}$ and the adjoint state $\bar{p}_{\alpha}$ needed for the convergence rates in the numerical realization of the problem.

We use here and in what follows the notation

$$
\begin{aligned}
& \|\cdot\|:=\|\cdot\|_{L^{2}(\Omega)}, \quad\|\cdot\|_{I}:=\|\cdot\|_{L^{2}\left(I, L^{2}(\Omega)\right)}, \\
& (\cdot, \cdot):=(\cdot, \cdot)_{L^{2}(\Omega)}, \quad \text { and } \quad(\cdot, \cdot)_{I}:=(\cdot, \cdot)_{L^{2}\left(I, L^{2}(\Omega)\right)} .
\end{aligned}
$$

Assumption 2.1. Let $y_{d} \in H^{2}\left(I, L^{2}(\Omega)\right) \cap H^{1}\left(I, H^{2}(\Omega) \cap H_{0}^{1}(\Omega)\right)$ with $\Delta y_{d}(T) \in H_{0}^{1}(\Omega)$ and $y_{0} \in H_{0}^{1}(\Omega)$. Furthermore, we expect $\Delta y_{0} \in H_{0}^{1}(\Omega)$. In the case of distributed controls, we assume $a, b \in H^{1}\left(I, L^{2}(\Omega)\right) \cap C\left(\bar{I}, H_{0}^{1}(\Omega) \cap C(\bar{\Omega})\right)$. In the case of located controls, we assume $g_{1} \in H_{0}^{1}(\Omega)$, and $a, b \in W^{1, \infty}(I)$.

Lemma 2.6 (Regularity of problem $(\mathbb{P}), \alpha>0$ ). Let Assumption 2.1 hold and let $\alpha>0$. For the unique solution $(\bar{y}, \bar{u})$ of $(\mathbb{P})$ and the corresponding adjoint state $\bar{p}$ there holds

- $\bar{p} \in H^{3}\left(I, L^{2}(\Omega)\right) \cap H^{2}\left(I, H^{2}(\Omega) \cap H_{0}^{1}(\Omega)\right) \hookrightarrow C^{2}\left(\bar{I}, H_{0}^{1}(\Omega)\right)$,

- $\bar{y} \in H^{2}\left(I, L^{2}(\Omega)\right) \cap H^{1}\left(I, H^{2}(\Omega) \cap H_{0}^{1}(\Omega)\right) \hookrightarrow C^{1}\left(\bar{I}, H_{0}^{1}(\Omega)\right), \quad$ and

- $\bar{u} \in W^{1, \infty}(I)$ in the case of located controls or

- $\bar{u} \in H^{1}\left(I, L^{2}(\Omega)\right) \cap C\left(\bar{I}, H_{0}^{1}(\Omega)\right) \cap C(\bar{I} \times \bar{\Omega})$ in the case of distributed controls.

With some constant $C>0$ independent of $\alpha$, we have the a priori estimates

$$
\begin{aligned}
&\left\|\partial_{t}^{2} \bar{y}\right\|_{I}+\left\|\partial_{t} \Delta \bar{y}\right\|_{I}+\max _{t \in[0, T]}\left\|\nabla \partial_{t} \bar{y}(t)\right\| \\
& \leq d_{1}(\bar{u}):=C\left(\|B \bar{u}\|_{H^{1}\left(I, L^{2}(\Omega)\right)}+\|\nabla B \bar{u}(0)\|+\left\|\nabla \Delta y_{0}\right\|\right) \\
&\left\|\partial_{t}^{2} \bar{p}\right\|_{I}+\left\|\partial_{t} \Delta \bar{p}\right\|_{I}+\max _{t \in[0, T]}\left\|\nabla \partial_{t} \bar{p}(t)\right\| \\
& \leq d_{0}(\bar{u}):=C\left(\left\|y_{d}\right\|_{H^{1}\left(I, L^{2}(\Omega)\right)}+\left\|\nabla y_{d}(T)\right\|+\|B \bar{u}\|_{I}+\left\|\nabla y_{0}\right\|\right), \\
&\left\|\partial_{t}^{3} \bar{p}\right\|_{I}+\left\|\partial_{t}^{2} \Delta \bar{p}\right\|_{I}+\max _{t \in[0, T]}\left\|\nabla \partial_{t}^{2} \bar{p}(t)\right\| \\
& \leq d_{1}^{+}(\bar{u}):=d_{1}(\bar{u})+C\left(\left\|\partial_{t}^{2} y_{d}\right\|_{I}+\left\|\nabla \partial_{t} y_{d}(T)\right\|+\left\|\nabla \Delta y_{d}(T)\right\|+\|\nabla B \bar{u}(T)\|\right) .
\end{aligned}
$$

Proof. See [12, Lemma 12].

Remark 2.2 (Regularity in the case $\alpha=0$ ). In the case $\alpha=0$, we have less regularity:

- $\bar{p} \in H^{1}\left(I, H^{2}(\Omega) \cap H_{0}^{1}(\Omega)\right) \cap H^{2}\left(I, L^{2}(\Omega)\right) \hookrightarrow C^{1}\left(\bar{I}, H_{0}^{1}(\Omega)\right)$, and

- $\bar{y} \in L^{2}\left(I, H^{2}(\Omega) \cap H_{0}^{1}(\Omega)\right) \cap H^{1}\left(I, L^{2}(\Omega)\right) \hookrightarrow C\left([0, T], H_{0}^{1}(\Omega)\right)$.

Since (2.10) does not hold, we can not derive regularity for $\bar{u}$ from that of $\bar{p}$ as above. We only know from the definition of $U_{\text {ad }}$ that $\bar{u} \in L^{\infty}\left(\Omega_{U}\right)$, but might be discontinuous as we will see later. 


\subsection{Tikhonov regularization}

For this subsection, it is useful to rewrite problem $(\mathbb{P})$ in the reduced form $\left(\mathbb{P}_{\alpha}\right)$ with $H:=L^{2}\left(I, L^{2}(\Omega)\right)$, fixed data $z:=y_{d}-S\left(0, y_{0}\right)$ and the linear and continuous control-tostate operator $T: U \rightarrow H, T u:=S(B u, 0)$. From now onwards we assume

$$
a \leq 0 \leq b
$$

in a pointwise almost everywhere sense where $a$ and $b$ are the bounds of the admissable set $U_{\text {ad }}$. For the limit problem $\left(\mathbb{P}_{0}\right)$, which we finally want to solve, this assumption can always be met by a simple transformation of the variables.

To prove rates of convergence with respect to $\alpha$, we rely on the following assumption.

Assumption 2.2. There exist a set $A \subset \Omega_{U}$, a function $w \in H$ with $T^{*} w \in L^{\infty}\left(\Omega_{U}\right)$, and constants $\kappa>0$ and $C \geq 0$, such that there holds the inclusion $\left\{x \in \Omega_{U} \mid B^{*} \bar{p}_{0}(x)=0\right\} \subset A^{c}$ for the complement $A^{c}=\Omega_{U} \backslash A$ of $A$ and in addition

1. (source condition)

$$
\chi_{A^{c}} \bar{u}_{0}=\chi_{A^{c}} P_{U_{\mathrm{ad}}}\left(T^{*} w\right) .
$$

2. $\left(\left(\bar{p}_{0^{-}}\right)\right.$measure condition $)$

$$
\forall \epsilon>0: \quad \operatorname{meas}\left(\left\{x \in A|0 \leq| B^{*} \bar{p}_{0}(x) \mid \leq \epsilon\right\}\right) \leq C \epsilon^{\kappa}
$$

with the convention that $\kappa:=\infty$ if the left-hand side of $(2.16)$ is zero for some $\epsilon>0$.

For a discussion of this assumption we refer to the texts subsequent to [17, Assumption 7] or [12, Assumption 15].

Key ingredient in the analysis of the regularization error and also of the discretization error considered later is the following lemma, see [17, Lemma 8] or [12, Lemma 16] for a proof.

Lemma 2.7. Let Assumption 2.2 .2 hold. For the solution $\bar{u}_{0}$ of $\left(\mathbb{P}_{0}\right)$, there holds with some constant $C>0$ independent of $\alpha$ and $u$

$$
C\left\|u-\bar{u}_{0}\right\|_{L^{1}(A)}^{1+1 / \kappa} \leq\left(B^{*} \bar{p}_{0}, u-\bar{u}_{0}\right)_{U} \quad \forall u \in U_{\mathrm{ad}}
$$

Using this lemma, we can now state regularization error estimates.

Theorem 2.1. For the regularization error there holds with positive constants $c$ and $C$ indepent of $\alpha>0$ the following.

1. Let Assumption 2.2 .2 be satisfied with meas $\left(A^{c}\right)=0$ (measure condition holds a.e. on the domain). Then the estimates

$$
\begin{aligned}
& \left\|\bar{u}_{\alpha}-\bar{u}_{0}\right\|_{L^{1}\left(\Omega_{U}\right)} \leq C \alpha^{\kappa}, \\
& \left\|\bar{u}_{\alpha}-\bar{u}_{0}\right\|_{U} \leq C \alpha^{\kappa / 2}, \\
& \left\|\bar{y}_{\alpha}-\bar{y}_{0}\right\|_{H} \leq C \alpha^{(\kappa+1) / 2}
\end{aligned}
$$

hold true. If $\kappa>1$ holds and in addition

$$
T^{*}: \operatorname{range}(T) \rightarrow L^{\infty}\left(\Omega_{U}\right) \quad \text { exists and is continuous, }
$$

we can improve (2.20) to

$$
\left\|\bar{y}_{\alpha}-\bar{y}_{0}\right\|_{H} \leq C \alpha^{\kappa}
$$


2. Let Assumption 2.2 be satisfied with meas $(A) \cdot \operatorname{meas}\left(A^{c}\right)>0$ (source and measure condition on parts of the domain). Then the following estimates

$$
\begin{aligned}
& \left\|\bar{u}_{\alpha}-\bar{u}_{0}\right\|_{L^{1}(A)} \leq C \alpha^{\min \left(\kappa, \frac{2}{1+1 / \kappa}\right)}, \\
& \left\|\bar{u}_{\alpha}-\bar{u}_{0}\right\|_{U} \leq C \alpha^{\min (\kappa, 1) / 2} \\
& \left\|\bar{y}_{\alpha}-\bar{y}_{0}\right\|_{H} \leq C \alpha^{\min ((\kappa+1) / 2,1)}
\end{aligned}
$$

hold true.

If furthermore $\kappa>1$ and (2.21) hold, we have the improved estimate

$$
\left\|\bar{u}_{\alpha}-\bar{u}_{0}\right\|_{L^{1}(A)} \leq C \alpha^{\kappa} .
$$

For a proof of this recent result, we refer to [17, Theorem 11] and [12, Theorem 19], where also a discussion can be found. We only recall two points for convenience here:

The assumption of the first case of the above Theorem implies

$$
\operatorname{meas}\left(\left\{x \in \Omega_{U} \mid B^{*} \bar{p}_{0}(x)=0\right\}\right)=0,
$$

which induces bang-bang controls, compare Remark 2.1.

By Lemma 2.6 and Remark 2.2 we can immediately see that the assumption (2.21) on $T^{*}$ is fulfilled for our parabolic problem.

\subsection{Bang-bang controls}

We now introduce a second measure condition which leads to an improved bound on the decay of smoothness in the derivative of the optimal control when $\alpha$ tends to zero. This bound will be useful later to derive improved convergence rates for the discretization errors.

Definition 2.1 ( $\bar{p}_{\alpha}$-measure condition). If for the set

$$
I_{\alpha}:=\left\{x \in \Omega_{U} \mid \alpha a<-B^{*} \bar{p}_{\alpha}<\alpha b\right\}
$$

the condition

$$
\exists \bar{\alpha}>0 \forall 0<\alpha<\bar{\alpha}: \quad \operatorname{meas}\left(I_{\alpha}\right) \leq C \alpha^{\kappa}
$$

holds true (with the convention that $\kappa:=\infty$ if the measure in $(2.29)$ is zero for all $0<\alpha<\bar{\alpha}$ ), we say that the $\bar{p}_{\alpha}$-measure condition is fulfilled.

Theorem 2.2. Let us assume the $\sigma$-condition

$$
\exists \sigma>0 \forall^{\prime} x \in \Omega_{U}: \quad a \leq-\sigma<0<\sigma \leq b .
$$

If the $\bar{p}_{\alpha}$-measure condition (2.29) is valid, then theorem 2.1.1 holds, omitting its first sentence ("Let Assumption...").

Proof. See [17, Theorem 15] or [12, Theorem 24].

If the limit problem is of certain regularity, both measure conditions coincide:

Corollary 2.1. Let a bang-bang solution be given, i.e., (2.27) holds true. In the case of $\kappa>1$, (2.21), and the $\sigma$-condition (2.30), both measure conditions are equivalent. 
Proof. See [17, Corollary 18] or [12, Corollary 27].

Let us now consider located controls. Since $\bar{p}_{\alpha} \in C^{1}\left(\bar{I}, L^{2}(\Omega)\right)$ for $\alpha \geq 0$ by Lemma 2.6 and Remark 2.2, we conclude

$$
\left\|\partial_{t} B^{*} \bar{p}_{\alpha}\right\|_{L^{\infty}(I)} \leq C\left\|\partial_{t} \bar{p}_{\alpha}\right\|_{L^{\infty}\left(I, L^{2}(\Omega)\right)} \leq C+C\left\|\bar{u}_{\alpha}\right\|_{U} \leq C
$$

with a constant $C>0$ independent of $\alpha$ due to the definition of $U_{\text {ad }}$. Recall that $a, b \in W^{1, \infty}(I)$ by Assumption 2.1. With this estimate, the projection formula (2.10) and the stability of the projection (see [12, Lemma 11]) we obtain the bound

$$
\left\|\partial_{t} \bar{u}_{\alpha}\right\|_{L^{\infty}(I)} \leq \frac{1}{\alpha}\left\|\partial_{t} B^{*} \bar{p}_{\alpha}\right\|_{L^{\infty}(I)}+\left\|\partial_{t} a\right\|_{L^{\infty}(I)}+\left\|\partial_{t} b\right\|_{L^{\infty}(I)} \leq C \frac{1}{\alpha}
$$

if $\alpha>0$ is sufficiently small.

If the $\bar{p}_{\alpha}$-measure condition (2.29) is valid, this decay of smoothness in terms of $\alpha$ can be relaxed in weaker norms, as the following Lemma shows.

Lemma 2.8 (Smoothness decay in the derivative). Let the $\bar{p}_{\alpha}$-measure condition (2.29) be fulfilled and located controls be given. Then for $\alpha>0$ sufficiently small there holds

$$
\left\|\partial_{t} \bar{u}_{\alpha}\right\|_{L^{p}(I)} \leq C \max \left(C_{a b}, \alpha^{\kappa / p-1}\right)
$$

with a constant $C>0$ independent of $\alpha$. Here, $C_{a b}:=\left\|\partial_{t} a\right\|_{L^{\infty}(I)}+\left\|\partial_{t} b\right\|_{L^{\infty}(I)}$ and $1 \leq p<\infty$. Note that $C_{a b}=0$ in the case of constant control bounds a and $b$.

Proof. See [17, Lemma 19] or [12, Lemma 28].

The question of necessity of Assumption 2.2 and the $\bar{p}_{\alpha}$-measure condition (2.28) to obtain the convergence rates of Theorem 2.1.1 is discussed in [17, sections 4 and 5] and [12, sections 1.4.3 and 1.4.4]. The results there show that in several cases the conditions are in fact necessary to obtain the convergence rates from above.

\section{The Discretized Problem}

\subsection{Discretization of the optimal control problem}

Consider a partition $0=t_{0}<t_{1}<\cdots<t_{M}=T$ of the time interval $\bar{I}$. With $I_{m}=$ $\left[t_{m-1}, t_{m}\right)$ we have $[0, T)=\bigcup_{m=1}^{M} I_{m}$. Furthermore, let $t_{m}^{*}=\left(t_{m-1}+t_{m}\right) / 2$ for $m=1, \ldots, M$ denote the interval midpoints. By $0=: t_{0}^{*}<t_{1}^{*}<\cdots<t_{M}^{*}<t_{M+1}^{*}:=T$ we get a second partition of $\bar{I}$, the so-called dual partition, namely $[0, T)=\bigcup_{m=1}^{M+1} I_{m}^{*}$, with $I_{m}^{*}=\left[t_{m-1}^{*}, t_{m}^{*}\right)$. The grid width of the first mentioned (primal) partition is given by the parameters $k_{m}=t_{m}-t_{m-1}$ and

$$
k=\max _{1 \leq m \leq M} k_{m} .
$$

Here and in what follows we assume $k<1$. We also denote by $k$ (in a slight abuse of notation) the grid itself.

We need the following conditions on sequences of time grids.

Assumption 3.1. There exist constants $0<\kappa_{1} \leq \kappa_{2}<\infty$ and $\mu>0$ independent of $k$ such that there holds

$$
\forall m \in\{1,2, \ldots, M-1\}: \kappa_{1} \leq \frac{k_{m}}{k_{m+1}} \leq \kappa_{2} \quad \text { and } \quad k \leq \mu \min _{m=1, \ldots, M} k_{m} .
$$


On these partitions of the time interval, we define the Ansatz and test spaces of the PetrovGalerkin schemes. These schemes will replace the continuous-in-time weak formulations of the state equation and the adjoint equation, i.e., (2.7) and (2.9), respectively. To this end, we define at first for an arbitrary Banach space $X$ the semidiscrete function spaces

$$
\begin{aligned}
P_{k}(X) & :=\left\{v \in C([0, T], X)|v|_{I_{m}} \in \mathcal{P}_{1}\left(I_{m}, X\right)\right\} \hookrightarrow H^{1}(I, X), \\
P_{k}^{*}(X): & =\left\{v \in C([0, T], X)|v|_{I_{m}^{*}} \in \mathcal{P}_{1}\left(I_{m}^{*}, X\right)\right\} \hookrightarrow H^{1}(I, X), \\
Y_{k}(X): & =\left\{v:[0, T] \rightarrow X^{*}|v|_{I_{m}} \in \mathcal{P}_{0}\left(I_{m}, X\right)\right\}
\end{aligned}
$$

Here, $\mathcal{P}_{i}(J, X), J \subset \bar{I}, i \in\{0,1\}$, is the set of polynomial functions in time with degree of at most $i$ on the interval $J$ with values in $X$. We note that functions in $P_{k}(X)$ can be uniquely determined by $M+1$ elements from $X$. The same holds true for functions $v \in Y_{k}(X)$ but with $v(T)$ only uniquely determined in $X^{*}$ by definition of the space. The reason for this is given in the discussion below $\left[12,(2.16)\right.$, p. 41]. Furthermore, for each function $v \in Y_{k}(X)$ we have $[v] \in L^{2}(I, X)$ where [.] denotes the equivalence class with respect to the almost-everywhere relation.

In the sequel, we will frequently use the following interpolation operators.

1. (Orthogonal projection) $\mathcal{P}_{Y_{k}(X)}: L^{2}(I, X) \rightarrow Y_{k}(X)$

$$
\left.\mathcal{P}_{Y_{k}(X)} v\right|_{I_{m}}:=\frac{1}{k_{m}} \int_{t_{m-1}}^{t_{m}} v d t, \quad m=1, \ldots, M, \quad \mathcal{P}_{Y_{k}(X)} v(T):=0 .
$$

2. (Piecewise linear interpolation on the dual grid) $\pi_{P_{k}^{*}(X)}: C([0, T], X) \cup Y_{k}(X) \rightarrow P_{k}^{*}(X)$

$$
\begin{aligned}
& \left.\pi_{P_{k}^{*}(X)} v\right|_{I_{1}^{*} \cup I_{2}^{*}}:=v\left(t_{1}^{*}\right)+\frac{t-t_{1}^{*}}{t_{2}^{*}-t_{1}^{*}}\left(v\left(t_{2}^{*}\right)-v\left(t_{1}^{*}\right)\right), \\
& \left.\pi_{P_{k}^{*}(X)} v\right|_{I_{m}^{*}}:=v\left(t_{m-1}^{*}\right)+\frac{t-t_{m-1}^{*}}{t_{m}^{*}-t_{m-1}^{*}}\left(v\left(t_{m}^{*}\right)-v\left(t_{m-1}^{*}\right)\right), \quad 3 \leq m \leq M-1, \\
& \left.\pi_{P_{k}^{*}(X)} v\right|_{I_{M}^{*} \cup I_{M+1}^{*}}:=v\left(t_{M-1}^{*}\right)+\frac{t-t_{M-1}^{*}}{t_{M}^{*}-t_{M-1}^{*}}\left(v\left(t_{M}^{*}\right)-v\left(t_{M-1}^{*}\right)\right) .
\end{aligned}
$$

The interpolation operators are obviously linear mappings. Furthermore, they are bounded, and we have error estimates, as [12, Lemma 31] shows.

In addition to the notation introduced after Remark 2.1, adding a subscript $I_{m}$ to a norm will indicate an $L^{2}\left(I_{m}, L^{2}(\Omega)\right)$ norm in the following. Inner products are treated in the same way.

Note that in all of the following results $C$ denotes a generic, strict positive real constant that does not depend on quantities which appear to the right or below of it.

Note that we can extend the bilinear form $A$ of (2.6) in its first argument to $W(I) \cup$ $Y_{k}\left(H_{0}^{1}(\Omega)\right)$, thus consider the operator

$$
A: W(I) \cup Y_{k}\left(H_{0}^{1}(\Omega)\right) \times W(I) \rightarrow \mathbb{R}, \quad A \text { given by }(2.6) .
$$

Using continuous piecewise linear functions in space, we can formulate fully discretized variants of the state and adjoint equation. 
We consider a regular triangulation $\mathcal{T}_{h}$ of $\Omega$ with mesh size

$$
h:=\max _{T \in \mathcal{T}_{h}} \operatorname{diam}(T),
$$

see, e.g., [19, Definition (4.4.13)], and $N=N(h)$ triangles. We assume that $h<1$. We also denote by $h$ (in a slight abuse of notation) the grid itself.

With the space

$$
X_{h}:=\left\{\phi_{h} \in C^{0}(\bar{\Omega})\left|\phi_{h}\right|_{T} \in \mathcal{P}_{1}(T, \mathbb{R}) \quad \forall T \in \mathcal{T}_{h}\right\}
$$

we define $X_{h 0}:=X_{h} \cap H_{0}^{1}(\Omega)$ to discretize $H_{0}^{1}(\Omega)$.

For the space grid we make use of a standard grid assumption, as we did for the time grid, sometimes called quasi-uniformity.

Assumption 3.2. There exists a constant $\mu>0$ independent of $h$ such that

$$
h \leq \mu \min _{T \in \mathcal{T}_{h}} \operatorname{diam}(T) .
$$

We fix fully discrete ansatz and test spaces, derived from their semidiscrete counterparts from (3.1), namely

$$
P_{k h}:=P_{k}\left(X_{h 0}\right), \quad P_{k h}^{*}:=P_{k h}^{*}\left(X_{h 0}\right), \quad \text { and } Y_{k h}:=Y_{k}\left(X_{h 0}\right) .
$$

With these spaces, we introduce fully discrete state and adjoint equations as follows.

Definition 3.1 (Fully discrete adjoint equation). For $h \in L^{2}\left(I, H^{-1}(\Omega)\right.$ ) find $p_{k h} \in P_{k h}$ such that

$$
A\left(\tilde{y}, p_{k h}\right)=\int_{0}^{T}\langle h(t), \tilde{y}(t)\rangle_{H^{-1}(\Omega) H_{0}^{1}(\Omega)} d t \quad \forall \tilde{y} \in Y_{k h} .
$$

Definition 3.2 (Fully discrete state equation). For $(f, g) \in L^{2}\left(I, H^{-1}(\Omega)\right) \times L^{2}(\Omega)$ find $y_{k h} \in Y_{k h}$, such that

$$
A\left(y_{k h}, v_{k h}\right)=\int_{0}^{T}\left\langle f(t), v_{k h}(t)\right\rangle_{H^{-1}(\Omega) H_{0}^{1}(\Omega)} d t+\left(g, v_{k h}(0)\right) \quad \forall v_{k h} \in P_{k h} .
$$

Existence and uniqueness of these two schemes follow as in the semidiscrete case discussed in [15] or [12, section 2.1.2].

Let us recall some stability results and error estimates for these schemes. The first result is $[12$, Lemma 56].

Lemma 3.1. Let $p_{k h} \in P_{k h}$ solve (3.7) with $h \in L^{2}\left(I, L^{2}(\Omega)\right)$. Then there exists a constant $C>0$ independent of the mesh sizes $k$ and $h$ such that

$$
\left\|p_{k h}\right\|_{H^{1}\left(I, L^{2}(\Omega)\right)}+\left\|\nabla p_{k h}\right\|_{C\left(\bar{I}, L^{2}(\Omega)\right)} \leq C\|h\|_{I} .
$$

For stability of a fully discrete state $y_{k h}$ and an error estimate, we recall [12, Lemma 59].

Lemma 3.2. Let $y$ be the solution of (2.7) for some $(f, g) \in L^{2}\left(I, H^{-1}(\Omega)\right) \times L^{2}(\Omega)$ and let $y_{k h} \in Y_{k h}$ be the solution of (3.8) for the same $(f, g)$. Then with a constant $C>0$ independent of $k$ and $h$, it holds

$$
\left\|y_{k h}\right\|_{I} \leq C\left(\|f\|_{L^{2}\left(I, H^{-1}(\Omega)\right)}+\|g\|\right)
$$


If furthermore the regularity $f \in L^{2}\left(I, L^{2}(\Omega)\right)$ as well as $g \in H_{0}^{1}(\Omega)$ is fulfilled, we have the error estimate

$$
\left\|y-y_{k h}\right\|_{I} \leq C\left(h^{2}+k\right)\left(\|f\|_{I}+\|\nabla g\|\right) .
$$

Let us now consider the error of the fully discrete adjoint state. We begin with an $L^{2}\left(I, L^{2}(\Omega)\right)$ norm result, which is [12, Lemma 62$]$.

Lemma 3.3. Let $p$ solve (2.9) for some right-hand side $h$ such that $p$ has the regularity $p \in$ $H^{1}\left(I, H^{2}(\Omega) \cap H_{0}^{1}(\Omega)\right) \cap H^{2}\left(I, L^{2}(\Omega)\right)$. Let furthermore $p_{k h} \in P_{k h}$ solve (3.7) for the same right-hand side $h$. Then it holds (with $h$ referring to the space grid)

$$
\left\|p_{k h}-p\right\|_{I} \leq C\left(k^{2}+h^{2}\right)\left(\left\|p_{t t}\right\|_{I}+\left\|\Delta p_{t}\right\|_{I}\right) .
$$

For the pointwise-in-time error, we recall [12, Lemma 65]:

Lemma 3.4. Let the assumptions of Lemma 3.3 be fulfilled. Then it holds

$$
\left\|p-p_{k h}\right\|_{L^{\infty}\left(I, L^{2}(\Omega)\right)} \leq C\left(h^{2}+k\right)\left(\left\|\Delta p_{t}\right\|_{I}+\left\|p_{t}\right\|_{L^{\infty}\left(I, L^{2}(\Omega)\right)}\right) .
$$

If in addition $p \in H^{2}\left(I, H^{2}(\Omega) \cap H_{0}^{1}(\Omega)\right)$ and $p_{t t} \in L^{\infty}\left(I, L^{2}(\Omega)\right)$ is known to hold, we have the improved estimate

$$
\begin{aligned}
& \left\|p-p_{k h}\right\|_{L^{\infty}\left(I, L^{2}(\Omega)\right)} \\
\leq & C\left(h^{2}+k^{2}\right)\left(\left\|\Delta p_{t}\right\|_{I}+\left\|p_{t}\right\|_{L^{\infty}\left(I, L^{2}(\Omega)\right)}\right)+C k^{2}\left(\left\|\Delta p_{t t}\right\|_{I}+\left\|p_{t t}\right\|_{L^{\infty}\left(I, L^{2}(\Omega)\right)}\right) .
\end{aligned}
$$

The following superconvergence result, which is [12, Lemma 66], will also be used in the later error analysis.

Lemma 3.5. Let $y \in Y$ and $y_{k h} \in Y_{k h}$ solve (2.7) and (3.8), respectively, with data $(f, g)$ fulfilling $f \in H^{1}\left(I, L^{2}(\Omega)\right), f(0) \in H_{0}^{1}(\Omega), g \in H_{0}^{1}(\Omega)$, and $\Delta g \in H_{0}^{1}(\Omega)$. By $p_{k h}(h) \in P_{k h}$ we denote the solution to (3.7) with right-hand side $h$. Then it holds

$$
\left\|y_{k h}-\mathcal{P}_{Y_{k}} y\right\|_{I}+\left\|p_{k h}\left(y_{k h}-y\right)\right\|_{C\left(\bar{I}, L^{2}(\Omega)\right)} \leq C\left(k^{2} F_{1}(f, g)+h^{2} F_{2}(f, g)\right)
$$

with

$$
\begin{aligned}
& F_{2}(f, g):=\|f\|_{I}+\|g\|_{H^{1}(\Omega)}, \\
& F_{1}(f, g):=F_{2}(f, g)+\left\|\partial_{t} f\right\|_{I}+\|f(0)\|_{H^{1}(\Omega)}+\|\Delta g\|_{H^{1}(\Omega)} .
\end{aligned}
$$

We are now able to introduce the discretized optimal control problem which reads

$$
\begin{aligned}
& \min _{y_{k h} \in Y_{k h}, u \in U_{\mathrm{ad}}} J\left(y_{k h}, u\right)=\min \frac{1}{2}\left\|y_{k h}-y_{d}\right\|_{I}^{2}+\frac{\alpha}{2}\|u\|_{U}^{2}, \\
& \text { s.t. } y_{k h}=S_{k h}\left(B u, y_{0}\right)
\end{aligned}
$$

where $\alpha, B, y_{0}, y_{d}$, and $U_{\text {ad }}$ are chosen as for $(\mathbb{P})$ and $S_{k h}$ is the solution operator associated to the fully discrete state equation (3.8). Recall that the space $Y_{k h}$ was introduced in (3.6).

For every $\alpha>0$, this problem admits a unique solution triple $\left(\bar{u}_{k h}, \bar{y}_{k h}, \bar{p}_{k h}\right)$ where $\bar{y}_{k h}=$ $S_{k h}\left(B \bar{u}_{k h}, y_{0}\right)$ and $\bar{p}_{k h}$ denotes the discrete adjoint state which is the solution of the fully 
discrete adjoint equation (3.7) with right-hand side $h:=\bar{y}_{k h}-y_{d}$. The first order necessary and sufficient optimality condition for problem $\left(\mathbb{P}_{k h}\right)$ is given by

$$
\bar{u}_{k h} \in U_{\mathrm{ad}}, \quad\left(\alpha \bar{u}_{k h}+B^{*} \bar{p}_{k h}, u-\bar{u}_{k h}\right)_{U} \geq 0 \quad \forall u \in U_{\mathrm{ad}},
$$

which can be rewritten as

$$
\bar{u}_{k h}=P_{U_{\text {ad }}}\left(-\frac{1}{\alpha} B^{*} \bar{p}_{k h}\right) .
$$

The above mentioned facts can be proven in the same way as for the continuous problem $(\mathbb{P})$.

Note that the control space $U$ is not discretized in the formulation $\left(\mathbb{P}_{k h}\right)$. In the numerical treatment, the relation (3.11) is instead exploited to get a discrete control. This approach is called Variational Discretization and was introduced in [13], see also [14, Chapter 3.2.5] for further details.

Remark 3.1. In the case $\alpha=0$, problem $\left(\mathbb{P}_{k h}\right)$ has at least one solution, but only $\bar{y}_{k h}$ and $\bar{p}_{k h}$ are unique, whereas an associated optimal control is in general non-unique. The reason is that $f \mapsto S_{k h}\left(f, y_{0}\right)$ is not injective in contrast to $f \mapsto S\left(f, y_{0}\right)$. However, the discrete solution is unique (and of bang-bang type) if the zero level set of $B^{*} \bar{p}_{k h}$ has measure zero.

\subsection{Error estimates for the regularized problem}

In what follows, we use the notation $y_{k h}(v):=S_{k h}\left(B v, y_{0}\right)$ with $v \in U_{\text {ad }}$, and $p_{k h}(h)$ is an abbreviation of the solution to (3.7) with right-hand side $h \in L^{2}\left(I, H^{-1}(\Omega)\right)$. Furthermore, $y(v)$ and $p(h)$ denote the continuous counterparts. Note that therefore we have $\bar{y}=y(\bar{u})$, $\bar{y}_{k h}=y_{k h}\left(\bar{u}_{k h}\right), \bar{p}=p\left(\bar{y}-y_{d}\right)$, and $\bar{p}_{k h}=p_{k h}\left(\bar{y}_{k h}-y_{d}\right)$.

The following Lemma provides a first step towards an error estimate with respect to the control and state discretization.

Lemma 3.6. Let $\bar{u}$ and $\bar{u}_{k h}$ solve $(\mathbb{P})$ and $\left(\mathbb{P}_{k h}\right)$, respectively, both for the same $\alpha \geq 0$. Then there holds

$$
\begin{aligned}
& \alpha\left\|\bar{u}_{k h}-\bar{u}\right\|_{U}^{2}+\left\|\bar{y}_{k h}-y_{k h}(\bar{u})\right\|_{I}^{2} \\
\leq & \left(B^{*}\left(p_{k h}\left(\bar{y}-y_{d}\right)-\bar{p}+p_{k h}\left(y_{k h}(\bar{u})-\bar{y}\right)\right), \bar{u}-\bar{u}_{k h}\right)_{U} .
\end{aligned}
$$

Proof. Inserting $\bar{u}_{k h}$ into (2.8) and $\bar{u}$ into (3.10) and adding up the resulting inequalities yields

$$
\left(\alpha\left(\bar{u}_{k h}-\bar{u}\right)+B^{*}\left(\bar{p}_{k h}-\bar{p}\right), \bar{u}_{k h}-\bar{u}\right)_{U} \leq 0
$$

After some simple manipulations we obtain

$$
\begin{gathered}
\alpha\left\|\bar{u}_{k h}-\bar{u}\right\|_{U}^{2} \leq\left(B^{*}\left(p_{k h}\left(\bar{y}-y_{d}\right)-\bar{p}+p_{k h}\left(y_{k h}(\bar{u})\right)-p_{k h}(\bar{y})\right), \bar{u}-\bar{u}_{k h}\right)_{U} \\
+\left(B^{*}\left(\bar{p}_{k h}-p_{k h}\left(y_{k h}(\bar{u})-y_{d}\right)\right), \bar{u}-\bar{u}_{k h}\right)_{U},
\end{gathered}
$$

and since the last line equals $-\left\|\bar{y}_{k h}-y_{k h}(\bar{u})\right\|_{I}^{2}$, we end up with the desired estimate by moving this term to the left.

We can now prove an error estimate, which resembles the standard estimate for variational discretized controls. It is build upon [15, Theorem 5.2]. Since we are interested in the limit behavior $\alpha \rightarrow 0$, we try to give a precise dependence of the right-hand side on $\alpha$. Note the splitting in terms of the quantities $d_{0}$ and $d_{1}$. In contrast to $d_{0}$, the term $d_{1}$ is not bounded if $\alpha \rightarrow 0$. 
Theorem 3.1. Let $\bar{u}$ and $\bar{u}_{k h}$ solve $(\mathbb{P})$ and $\left(\mathbb{P}_{k h}\right)$, respectively, both for the same $\alpha \geq 0$. Then there exists a constant $\alpha_{\max }>0$ independent of $k$ and $h$, so that for all $0 \leq \alpha \leq \alpha_{\max }$ (with the convention " $1 / 0=\infty=d_{1}$ " in the case of $\alpha=0$ ) the estimate

$$
\begin{aligned}
& \sqrt{\alpha}\left\|\bar{u}_{k h}-\bar{u}\right\|_{U}+\left\|\bar{y}_{k h}-y_{k h}(\bar{u})\right\|_{I} \\
\leq & C \min \left(\frac{k^{2}+h^{2}}{\sqrt{\alpha}} d_{0},(k+h) \sqrt{\left\|\bar{u}_{k h}-\bar{u}\right\|_{U}} \sqrt{d_{0}}\right)+C \min \left(k^{2} d_{1}, k d_{0}\right)+C h^{2} d_{0} \\
\leq & C \max \left(d_{0}+1, \sqrt{d_{0}}\right) \min \left(\frac{k^{2}}{\alpha}+\frac{h^{2}}{\sqrt{\alpha}}, k+h\right)
\end{aligned}
$$

is satisfied with the constants $d_{0}=d_{0}(\bar{u})$ and $d_{1}=d_{1}(\bar{u})$ from the estimates (2.13) in Lemma 2.6 .

Proof. We split the right-hand side of the estimate from Lemma 3.6 and get with the Cauchy-Schwarz inequality

$$
\begin{aligned}
& \alpha\left\|\bar{u}_{k h}-\bar{u}\right\|_{U}^{2}+\left\|\bar{y}_{k h}-y_{k h}(\bar{u})\right\|_{I}^{2} \\
\leq & \left\|p_{k h}\left(\bar{y}-y_{d}\right)-\bar{p}\right\|_{I}\left\|\bar{u}-\bar{u}_{k h}\right\|_{U}+\left(B^{*}\left(p_{k h}\left(y_{k h}(\bar{u})-\bar{y}\right)\right), \bar{u}-\bar{u}_{k h}\right)_{U}=: I+I I .
\end{aligned}
$$

With the help of Lemmas 3.3 and 2.6, we conclude

$$
\left\|p_{k h}\left(\bar{y}-y_{d}\right)-\bar{p}\right\|_{I} \leq C\left(k^{2}+h^{2}\right)\left(\left\|\bar{p}_{t t}\right\|_{I}+\left\|\Delta \bar{p}_{t}\right\|_{I}\right) \leq C\left(k^{2}+h^{2}\right) d_{0}
$$

Now we use Cauchy's inequality to obtain

$$
I \leq \frac{C}{\alpha}\left\|p_{k h}\left(\bar{y}-y_{d}\right)-\bar{p}\right\|_{I}^{2}+\frac{\alpha}{2}\left\|\bar{u}-\bar{u}_{k h}\right\|_{U}^{2} .
$$

Here, the second addend can be moved to the left. Both estimates can be summarized as

$$
\sqrt{I} \leq C \min \left(\frac{k^{2}+h^{2}}{\sqrt{\alpha}} d_{0},(k+h) \sqrt{\left\|\bar{u}_{k h}-\bar{u}\right\|_{U}} \sqrt{d_{0}}\right) .
$$

The addend $I I$ can be estimated as

$$
I I=\left(y_{k h}(\bar{u})-\bar{y}, y_{k h}(\bar{u})-\bar{y}_{k h}\right)_{I} \leq \frac{1}{2}\left(\left\|y_{k h}(\bar{u})-\bar{y}\right\|_{I}^{2}+\left\|y_{k h}(\bar{u})-\bar{y}_{k h}\right\|_{I}^{2}\right) .
$$

We move the second term to the left. Note that in the previous estimate $\bar{y}$ can be replaced by $\mathcal{P}_{Y_{k}} \bar{y}$ by definition of $\mathcal{P}_{Y_{k}}$. We thus can invoke either the error estimate of the state equation (3.9) from Lemma 3.2 or the superconvergence result from Lemma 3.5. In conclusion, we have

$$
\sqrt{I I} \leq C \min \left(\left(k+h^{2}\right) d_{0}, k^{2} d_{1}+h^{2} d_{0}\right)=\min \left(k d_{0}, k^{2} d_{1}\right)+h^{2} d_{0} .
$$

Together with the estimate for $\sqrt{I}$, we obtain the first inequality of the claim.

For the second inequality, we first note that with the help of the projection formula (2.10), the stability of the projection, see, e.g., [12, Lemma 11], and the regularity result [12, Lemma 6$]$ one immediately derives the estimate

$$
\begin{aligned}
& \|\bar{u}\|_{H^{1}(I, \tilde{U})}+\|B \bar{u}(0)\|_{H^{1}(\Omega)} \\
\leq & \frac{C}{\alpha}\left(\|\bar{p}\|_{H^{1}\left(I, L^{2}(\Omega)\right)}+\|\bar{p}(0)\|_{H^{1}(\Omega)}\right)+C(a)+C(b) \\
\leq & \frac{C}{\alpha}\left(\left\|y_{d}\right\|_{I}+\|\bar{u}\|_{U}+\left\|y_{0}\right\|_{H^{1}(\Omega)}\right)+C(a)+C(b),
\end{aligned}
$$


where $\tilde{U} \in\left\{\mathbb{R}, L^{2}(\Omega)\right\}$, depending on whether located or distributed controls are given, and $C(x)=\|x\|_{H^{1}(I, \tilde{U})}+\|x(0)\|_{X}$ with $X=H^{1}(\Omega)$ (distributed controls) or $X=\mathbb{R}$ (located controls). This term is bounded due to Assumption 2.1.

Since there exists an $\alpha_{\max }>0$, depending only on the data $a, b, y_{0}, y_{d}$, such that

$$
\forall 0 \leq \alpha \leq \alpha_{\max }: \quad d_{1}+d_{1}^{+} \leq C \frac{1}{\alpha}\left(d_{0}+1\right)
$$

holds with $d_{1}^{+}:=d_{1}^{+}(\bar{u})$ from the estimates (2.13) in Lemma 2.6, and since $\sqrt{\left\|\bar{u}_{k h}-\bar{u}\right\|_{U}}$ is bounded independently of $\alpha$ due to the definition of $U_{\mathrm{ad}}$, we get the claim.

From the proof of the previous theorem, one can immediately derive a first robust (with respect to $\alpha \rightarrow 0$ ) error bound for the optimal state.

Corollary 3.1. Let $\bar{u}$ and $\bar{u}_{k h}$ solve $(\mathbb{P})$ and $\left(\mathbb{P}_{k h}\right)$, respectively, both for the same arbitrarily chosen $\alpha \geq 0$. Then there holds

$$
\left\|\bar{y}-\bar{y}_{k h}\right\|_{I} \leq C(k+h) \max \left(d_{0}+1, \sqrt{d_{0}}\right)
$$

with a constant $C>0$ independent of $\alpha$ where $d_{0}$ is given in Theorem 3.1.

Proof. Combining

$$
\left\|\bar{y}-\bar{y}_{k h}\right\|_{I} \leq\left\|y_{k h}(\bar{u})-\bar{y}_{k h}\right\|_{I}+\left\|\bar{y}-y_{k h}(\bar{u})\right\|_{I}
$$

with the previous Theorem and (3.9) from Lemma 3.2 proves the claim.

Now, from the above Theorem we derive further non-robust estimates for the discrete state and adjoint state. Finally, we prove second order convergence for $\pi_{P_{k}^{*}} \bar{y}_{k h}$, i.e., the piecewise linear interpolation on the dual grid of the optimal state. This function is obtained for free from $\bar{y}_{k h}$, since $\bar{y}_{k h}$ only has to be evaluated on the dual time grid. Compare [15, Theorem 5.3] for the convergence of the interpolation in the semidiscrete case.

Corollary 3.2. Let $\bar{u}$ and $\bar{u}_{k h}$ denote the solutions to $(\mathbb{P})$ and $\left(\mathbb{P}_{k h}\right)$, respectively, both for the same sufficiently small $\alpha>0$ (in the sense of Theorem 3.1). With $d_{0}$ and $d_{1}$ as in Theorem 3.1 and

$$
d_{1}^{+}:=d_{1}^{+}(\bar{u})=d_{1}(\bar{u})+C\left(\left\|\partial_{t}^{2} y_{d}\right\|_{I}+\left\|\nabla \partial_{t} y_{d}(T)\right\|+\left\|\nabla \Delta y_{d}(T)\right\|+\|\nabla B \bar{u}(T)\|\right)
$$

from the estimates (2.13) in Lemma 2.6, the estimates

$$
\begin{gathered}
\left\|\bar{u}-\bar{u}_{k h}\right\|_{U} \leq C\left(\frac{k^{2} d_{1}}{\sqrt{\alpha}}+\frac{k^{2}+h^{2}}{\alpha} d_{0}\right) \leq C\left(\frac{k^{2}}{\alpha^{3 / 2}}+\frac{h^{2}}{\alpha}\right)\left(d_{0}+1\right), \\
\left\|\bar{y}-\bar{y}_{k h}\right\|_{I} \leq C\left(k+\frac{k^{2}}{\alpha}+\frac{h^{2}}{\sqrt{\alpha}}\right)\left(d_{0}+1\right), \\
\alpha\left\|\bar{u}-\bar{u}_{k h}\right\|_{L^{\infty}(I, \tilde{U})}+\left\|\bar{p}-\bar{p}_{k h}\right\|_{L^{\infty}\left(I, L^{2}(\Omega)\right)}+\left\|\bar{y}-\pi_{P_{k}^{*}} \bar{y}_{k h}\right\|_{I} \\
\leq C\left(k^{2} d_{1}^{+}+\frac{k^{2}+h^{2}}{\sqrt{\alpha}} d_{0}\right) \leq C\left(\frac{k^{2}}{\alpha}+\frac{h^{2}}{\sqrt{\alpha}}\right)\left(d_{0}+1\right)
\end{gathered}
$$

hold with $\tilde{U} \in\left\{\mathbb{R}, L^{2}(\Omega)\right\}$ depending on whether located or distributed controls are given. 
Proof. The first estimate for the optimal control and the estimate for the optimal state follow from Theorem 3.1. For the latter, we argue as in the proof of Corollary 3.1.

For the optimal adjoint state, we split the error into three parts to obtain with $L:=$ $L^{\infty}\left(I, L^{2}(\Omega)\right)$

$$
\left\|\bar{p}-\bar{p}_{k h}\right\|_{L} \leq\left\|\bar{p}-p_{k h}\left(\bar{y}-y_{d}\right)\right\|_{L}+\left\|p_{k h}\left(\mathcal{P}_{Y_{k}} \bar{y}-y_{k h}(\bar{u})\right)\right\|_{L}+\left\|p_{k h}\left(y_{k h}(\bar{u})-\bar{y}_{k h}\right)\right\|_{L} .
$$

With the second error estimate from Lemma 3.4, the regularity given in Lemma 2.6, and the estimate from Lemma 3.5, we conclude

$$
\left\|\bar{p}-p_{k h}\left(\bar{y}-y_{d}\right)\right\|_{L}+\left\|p_{k h}\left(\mathcal{P}_{Y_{k}} \bar{y}-y_{k h}(\bar{u})\right)\right\|_{L} \leq C\left(h^{2} d_{0}+k^{2} d_{1}^{+}\right)
$$

since $d_{1} \leq d_{1}^{+}$.

Stability from Lemma 3.1 combined with Theorem 3.1 gives the estimate

$$
\left\|p_{k h}\left(y_{k h}(\bar{u})-\bar{y}_{k h}\right)\right\|_{L} \leq C \frac{k^{2}+h^{2}}{\sqrt{\alpha}} d_{0}+C k^{2} d_{1}+C h^{2} d_{0} .
$$

From this, we get

$$
\left\|\bar{p}-\bar{p}_{k h}\right\|_{L} \leq C \frac{k^{2}+h^{2}}{\sqrt{\alpha}} d_{0}+C k^{2} d_{1}^{+} .
$$

The projection formulae (2.10) and (3.11), Lipschitz continuity of the projection given in [12, Lemma 11], and stability of $B^{*}$ yield

$$
\left\|\bar{u}-\bar{u}_{k h}\right\|_{L^{\infty}(I, \tilde{U})} \leq C \frac{1}{\alpha}\left\|\bar{p}-\bar{p}_{k h}\right\|_{L} .
$$

Together with the just established estimate this yields the pointwise-in-time error estimate for the optimal control.

For the proof of the error $\left\|\bar{y}-\pi_{P_{k}^{*}} \bar{y}_{k h}\right\|_{I}$, we refer the reader to [12, Corollary 71].

Using the inequality (3.14), we can finally reduce the non-robust constants $d_{1}$ and $d_{1}^{+}$to the robust one $d_{0}$.

Let us comment on the estimates of Theorem 3.1 and Corollary 3.2. These estimates show that if $\alpha>0$ is fixed, we have convergence rates $h^{2}+k^{2}$ except for the state error. Invoking the regularization error, one obtains estimates for the total error between the limit problem and the discrete regularized one. From this, a coupling rule for the parameters $\alpha, k$ and $h$ can be derived.

As an example, consider the error in the projected state for the special case $\kappa=1$. With the help of Theorem 2.1, and Corollary 3.2 we get with the inequality (3.14) the estimate

$$
\begin{aligned}
& \left\|\bar{y}_{0}-\pi_{P_{k}^{*}}\left(\bar{y}_{k h}\right)\right\|_{I} \leq\left\|\bar{y}_{0}-\bar{y}_{\alpha}\right\|_{I}+\left\|\bar{y}_{\alpha}-\pi_{P_{k}^{*}}\left(\bar{y}_{k h}\right)\right\|_{I} \\
\leq & C\left(\alpha+k^{2} d_{1}^{+}+\frac{k^{2}+h^{2}}{\sqrt{\alpha}} d_{0}\right) \leq C\left(\alpha+\frac{k^{2}}{\alpha}+\frac{h^{2}}{\sqrt{\alpha}}\right)\left(d_{0}+1\right),
\end{aligned}
$$

which implies $\left\|\bar{y}_{0}-\pi_{P_{k}^{*}}\left(\bar{y}_{k h}\right)\right\|_{I} \leq C k=C h^{4 / 3}$ when setting $\alpha=k=h^{4 / 3}$.

However, if the decay estimate $d_{1}^{+} \leq \frac{C}{\alpha}$, i.e., (3.14), can be improved, we can get a better convergence rate (with respect to $k$ ) for the total error. In Lemma 2.8 we saw that this is indeed possible.

Unfortunately, space convergence of order $h^{2}$ is not achievable in the above mentioned estimates if $\alpha$ tends to zero due to $\alpha$ appearing in the denominator. To overcome this, we establish other estimates in the next subsection. The question of improving the decay estimate (3.14) is discussed in the next but one subsection using the estimates of the next subsection. 


\subsection{Robust error estimates}

All the previous estimates (except Corollary 3.1) are not robust for $\alpha \rightarrow 0$, since $\alpha$ appears always in a denominator on the right-hand side. Especially, convergence of order $h^{2}$ is not achievable as discussed at the end of the previous subsection. With some refined analysis, however, one can show estimates which are robust with respect to $\alpha \rightarrow 0$. A key ingredient is Lemma 2.7, which was also very important for the derivation of the regularization error.

Recall the notation from the beginning of subsection 3.2 .

Theorem 3.2. Let Assumption 2.2 be fulfilled so that either (2.18) or (2.23) from Theorem 2.1 holds. We denote the valid convergence rate for the control by $\alpha^{\omega_{1}}$. Then, either (2.20) or (2.25) is fulfilled. We abbreviate the corresponding convergence rate by $\alpha^{\omega_{2}}$.

Let $\bar{u}_{0}$ be the solution of $\left(\mathbb{P}_{0}\right)$ with associated state $\bar{y}_{0}$. For some $\alpha \geq 0$ let in addition $\bar{u}_{d}:=\bar{u}_{\alpha, k h} \in U_{\mathrm{ad}}$ be a solution of $\left(\mathbb{P}_{k h}\right)$ with associated discrete state $\bar{y}_{d}$ and adjoint state $\bar{p}_{d}$. Then there holds

$$
\begin{aligned}
& \left\|\bar{u}_{0}-\bar{u}_{d}\right\|_{L^{1}(A)} \\
& \leq C\left(\alpha^{\omega_{1}}+\left\|B^{*}\left(p_{k h}-p\right)\left(y\left(\bar{u}_{d}\right)-y_{d}\right)\right\|_{L^{\infty}(A)}^{\kappa}+\left\|B^{*}\left(p_{k h}-p\right)\left(y\left(\bar{u}_{d}\right)-y_{d}\right)\right\|_{L^{1}\left(A^{c}\right)}^{\frac{1}{1+1 / \kappa}}\right. \\
& \left.\quad+\left\|B^{*} p_{k h}\left(y_{k h}\left(\bar{u}_{d}\right)-y\left(\bar{u}_{d}\right)\right)\right\|_{L^{\infty}(A)}^{\kappa}+\left\|B^{*} p_{k h}\left(y_{k h}\left(\bar{u}_{d}\right)-y\left(\bar{u}_{d}\right)\right)\right\|_{L^{1}\left(A^{c}\right)}^{\frac{1}{1+1 / \kappa}}\right)
\end{aligned}
$$

for the error in the control and

$$
\begin{aligned}
\left\|\bar{y}_{0}-\bar{y}_{d}\right\|_{I} \leq C & \left(\alpha^{\omega_{2}}+\left\|B^{*}\left(p_{k h}-p\right)\left(y\left(\bar{u}_{d}\right)-y_{d}\right)\right\|_{L^{\infty}(A)}^{\frac{1+\kappa}{2}}\right. \\
& +\left\|B^{*}\left(p_{k h}-p\right)\left(y\left(\bar{u}_{d}\right)-y_{d}\right)\right\|_{L^{1}\left(A^{c}\right)}^{1 / 2}+\left\|B^{*} p_{k h}\left(y_{k h}\left(\bar{u}_{d}\right)-y\left(\bar{u}_{d}\right)\right)\right\|_{L^{\infty}(A)}^{\frac{1+\kappa}{2}} \\
& \left.+\left\|B^{*} p_{k h}\left(y_{k h}\left(\bar{u}_{d}\right)-y\left(\bar{u}_{d}\right)\right)\right\|_{L^{1}\left(A^{c}\right)}^{1 / 2}+\left\|y_{k h}\left(\bar{u}_{d}\right)-y\left(\bar{u}_{d}\right)\right\|_{I}\right)
\end{aligned}
$$

for the error in the state.

Proof. To the estimate (2.17) from Lemma 2.7 with $u:=\bar{u}_{d}$, i.e.,

$$
C\left\|\bar{u}_{d}-\bar{u}_{0}\right\|_{L^{1}(A)}^{1+1 / \kappa} \leq\left(-B^{*} \bar{p}_{0}, \bar{u}_{0}-\bar{u}_{d}\right)_{U},
$$

we add the necessary condition $(3.10)$ for $\bar{u}_{d}$ with $u:=\bar{u}_{0}$, which can be rewritten as

$$
\alpha\left\|\bar{u}_{0}-\bar{u}_{d}\right\|_{U}^{2} \leq\left(\alpha \bar{u}_{0}+B^{*} \bar{p}_{d}, \bar{u}_{0}-\bar{u}_{d}\right)_{U} .
$$

We end up with

$$
\begin{aligned}
& \left\|\bar{u}_{0}-\bar{u}_{d}\right\|_{L^{1}(A)}^{1+1 / \kappa}+\alpha\left\|\bar{u}_{0}-\bar{u}_{d}\right\|_{U}^{2}+\left\|y\left(\bar{u}_{0}\right)-y\left(\bar{u}_{d}\right)\right\|_{I}^{2} \\
\leq & C\left(-B^{*} p\left(y\left(\bar{u}_{d}\right)-y_{d}\right)+B^{*} p_{k h}\left(y_{k h}\left(\bar{u}_{d}\right)-y_{d}\right)+\alpha \bar{u}_{0}, \bar{u}_{0}-\bar{u}_{d}\right)_{U} \\
\leq & C(\underbrace{B^{*}\left(p_{k h}-p\right)\left(y\left(\bar{u}_{d}\right)-y_{d}\right)}_{I}+\underbrace{B^{*} p_{k h}\left(y_{k h}\left(\bar{u}_{d}\right)-y\left(\bar{u}_{d}\right)\right)}_{I I}+\underbrace{\alpha \bar{u}_{0}}_{I I I}, \bar{u}_{0}-\bar{u}_{d})_{U} .
\end{aligned}
$$

We now use [12, Lemma 18], Cauchy's and Young's inequality to estimate III as

$$
\begin{aligned}
& \alpha\left(\bar{u}_{0}, \bar{u}_{0}-\bar{u}_{d}\right)_{U} \leq \alpha C\left(\left\|T\left(\bar{u}_{d}-\bar{u}_{0}\right)\right\|_{H}+\left\|\bar{u}_{d}-\bar{u}_{0}\right\|_{L^{1}(A)}\right) \\
\leq & C \alpha^{2}+\frac{1}{4}\left\|T\left(\bar{u}_{d}-\bar{u}_{0}\right)\right\|_{H}^{2}+C \alpha^{1+\kappa}+\frac{1}{4}\left\|\bar{u}_{d}-\bar{u}_{0}\right\|_{L^{1}(A)}^{1+1 / \kappa} .
\end{aligned}
$$


The $\alpha$-free terms can now be moved to the left, since $\left\|T\left(\bar{u}_{d}-\bar{u}_{0}\right)\right\|_{H}=\left\|y\left(\bar{u}_{d}\right)-y\left(\bar{u}_{0}\right)\right\|_{I}$. Note that $C \alpha^{2}$ can be omitted if $A=\Omega_{U}$ since by Young's inequality we then get

$$
\alpha\left\|\bar{u}_{\alpha}-\bar{u}_{0}\right\|_{L^{1}\left(\Omega_{U}\right)} \leq C \alpha^{\kappa+1}+C\left\|\bar{u}_{\alpha}-\bar{u}_{0}\right\|_{L^{1}\left(\Omega_{U}\right)}^{1+1 / \kappa} .
$$

Thus only the term $C \alpha^{2 \omega_{2}}$ remains on the right-hand side.

For $I$ and $I I$, we proceed with the help of Young's inequality to obtain

$$
\begin{aligned}
& \left(\sim, \bar{u}_{0}-\bar{u}_{d}\right)_{U} \\
= & \left(\sim, \bar{u}_{0}-\bar{u}_{d}\right)_{L^{2}(A)}+\left(\sim, \bar{u}_{0}-\bar{u}_{d}\right)_{L^{2}\left(A^{c}\right)} \\
\leq & C\|\sim\|_{L^{\infty}(A)}^{1+\kappa}+\frac{1}{4}\left\|\bar{u}_{0}-\bar{u}_{d}\right\|_{L^{1}(A)}^{1+1 / \kappa}+\|\sim\|_{L^{1}\left(A^{c}\right)}\|b-a\|_{L^{\infty}\left(A^{c}\right)}
\end{aligned}
$$

and move the second addend to the left. Finally, we end up with

$$
\begin{aligned}
& \left\|\bar{u}_{0}-\bar{u}_{d}\right\|_{L^{1}(A)}^{1+1 / \kappa}+\alpha\left\|\bar{u}_{0}-\bar{u}_{d}\right\|_{U}^{2}+\left\|y\left(\bar{u}_{0}\right)-y\left(\bar{u}_{d}\right)\right\|_{I}^{2} \\
\leq & C\left(\alpha^{2 \omega_{2}}+\left\|B^{*}\left(p_{k h}-p\right)\left(y\left(\bar{u}_{d}\right)-y_{d}\right)\right\|_{L^{\infty}(A)}^{1+\kappa}+\left\|B^{*}\left(p_{k h}-p\right)\left(y\left(\bar{u}_{d}\right)-y_{d}\right)\right\|_{L^{1}\left(A^{c}\right)}\right. \\
& \left.+\left\|B^{*} p_{k h}\left(y_{k h}\left(\bar{u}_{d}\right)-y\left(\bar{u}_{d}\right)\right)\right\|_{L^{\infty}(A)}^{1+\kappa}+\left\|B^{*} p_{k h}\left(y_{k h}\left(\bar{u}_{d}\right)-y\left(\bar{u}_{d}\right)\right)\right\|_{L^{1}\left(A^{c}\right)}\right) .
\end{aligned}
$$

From this we conclude the claim for the optimal control.

The just established estimate together with the decomposition

$$
\left\|\bar{y}_{0}-\bar{y}_{d}\right\|_{I} \leq\left\|y_{k h}\left(\bar{u}_{d}\right)-y\left(\bar{u}_{d}\right)\right\|_{I}+\left\|y\left(\bar{u}_{d}\right)-y\left(\bar{u}_{0}\right)\right\|_{I}
$$

yields the claim for the optimal state.

Remark 3.2. The error estimate (3.16) in the previous Theorem for $\alpha>0$ is also valid if $\bar{u}_{0}$ is replaced by $\bar{u}_{\alpha}$, i.e., the solution of $(\mathbb{P})$ for some $\alpha>0$, since by Theorem 2.1 we can estimate

$$
\begin{aligned}
& \left\|\bar{u}_{\alpha}-\bar{u}_{d}\right\|_{L^{1}(A)} \\
\leq & \left\|\bar{u}_{\alpha}-\bar{u}_{0}\right\|_{L^{1}(A)}+\left\|\bar{u}_{0}-\bar{u}_{d}\right\|_{L^{1}(A)} \leq C \alpha^{\omega_{1}}+\left\|\bar{u}_{0}-\bar{u}_{d}\right\|_{L^{1}(A)} .
\end{aligned}
$$

Likewise, in (3.17) the state $\bar{y}_{0}$ can be replaced by $\bar{y}_{\alpha}$.

We will make use of this fact in the proof of the next theorem.

In combination with the error estimates for the state and adjoint state equations previously derived, we can now prove a first error estimate between solutions of $\left(\mathbb{P}_{k h}\right)$ and $\left(\mathbb{P}_{0}\right)$, which is robust if $\alpha$ tends to zero. In view of the numerical verification, we restrict ourselves now to the situation $A=\Omega_{U}$ and located controls.

Theorem 3.3. Let the assumptions of Theorem 3.2 be fulfilled. Further, we assume located controls and $A=\Omega_{U}$ (measure condition on the whole domain). Then there hold the estimates

$$
\left\|\bar{u}_{0}-\bar{u}_{d}\right\|_{U}^{2}+\left\|\bar{u}_{0}-\bar{u}_{d}\right\|_{L^{1}\left(\Omega_{U}\right)} \leq C\left(\alpha+h^{2}+k\right)^{\kappa}\left(1+d_{0}\left(\bar{u}_{d}\right)^{\kappa}\right)
$$

for the error in the control, for the auxiliary error

$$
\left\|\bar{y}_{d}-y_{k h}\left(\bar{u}_{\alpha}\right)\right\|_{I}^{2} \leq C\left(h^{2}+k\right) d_{0}\left(\bar{u}_{\alpha}\right)\left(\alpha^{\kappa}+\left(h^{2}+k\right)^{\kappa} d_{0}\left(\bar{u}_{d}\right)^{\kappa}\right)
$$


where by $\bar{u}_{\alpha}$ we denote the solution of $(\mathbb{P})$, and

$$
\left\|\bar{y}_{d}-\bar{y}_{0}\right\|_{I} \leq C\left(\alpha^{\frac{1+\kappa}{2}}+\left(h^{2}+k\right)^{\min \left(1, \frac{1+\kappa}{2}\right)}\right)\left(1+d_{0}\left(\bar{u}_{d}\right)^{\min \left(1, \frac{1+\kappa}{2}\right)}\right)
$$

for the error in the state.

If $\kappa>1$, we have the improved convergence rate

$$
\left\|\bar{y}_{d}-\bar{y}_{0}\right\|_{I} \leq C\left(\alpha^{\kappa}+h^{2}+k\right)\left(1+\max \left(d_{0}\left(\bar{u}_{d}\right)^{\kappa}, d_{0}\left(\bar{u}_{\alpha}\right)\right)\right),
$$

thus observe the regularization error (2.22).

Proof. Combining Theorem 3.2 with the adjoint error estimate in Lemma 3.4, the adjoint stability from Lemma 3.1, the error estimate (3.9) in Lemma 3.2, and the regularity given in Lemma 2.6 and Remark 2.2, we achieve (3.22) and (3.24) except for the $U$ error in the control. This error can be derived from the corresponding $L^{1}$ error by the estimate

$$
\begin{aligned}
\left\|\bar{u}_{0}-\bar{u}_{d}\right\|_{U}^{2} & \leq\left\|\bar{u}_{0}-\bar{u}_{d}\right\|_{L^{\infty}\left(\Omega_{U}\right)}\left\|\bar{u}_{0}-\bar{u}_{d}\right\|_{L^{1}\left(\Omega_{U}\right)} \\
& \leq\|b-a\|_{L^{\infty}\left(\Omega_{U}\right)}\left\|\bar{u}_{0}-\bar{u}_{d}\right\|_{L^{1}\left(\Omega_{U}\right)},
\end{aligned}
$$

which follows immediately from standard $L^{p}$ interpolation, see, e.g., [20, Theorem 2.11], and the definition of $U_{\text {ad }}$.

Let us now tackle the improved state convergence, thereby proving the estimate (3.23). We split the error into three parts and obtain with the help of (2.22) and the error estimate (3.9) from Lemma 3.2

$$
\begin{aligned}
& \left\|\bar{y}_{d}-\bar{y}_{0}\right\|_{I}^{2} \\
\leq & C\left(\left\|\bar{y}_{d}-y_{k h}\left(\bar{u}_{\alpha}\right)\right\|_{I}^{2}+\left\|y_{k h}\left(\bar{u}_{\alpha}\right)-y\left(\bar{u}_{\alpha}\right)\right\|_{I}^{2}+\left\|y\left(\bar{u}_{\alpha}\right)-y\left(\bar{u}_{0}\right)\right\|_{I}^{2}\right) \\
\leq & C\left(\left\|\bar{y}_{d}-y_{k h}\left(\bar{u}_{\alpha}\right)\right\|_{I}^{2}+\left(h^{2}+k\right)^{2} d_{0}^{2}\left(\bar{u}_{\alpha}\right)+\alpha^{2 \kappa}\right),
\end{aligned}
$$

where we also used (2.13) from Lemma 2.6.

For the remaining term, we invoke Lemma 3.6 in combination with (3.16) and Remark 3.2 and setting $L:=L^{\infty}\left(I, L^{2}(\Omega)\right)$ we obtain with the stability of $B^{*}$ for located controls

$$
\begin{aligned}
& \left\|\bar{y}_{d}-y_{k h}\left(\bar{u}_{\alpha}\right)\right\|_{I}^{2} \\
\leq & C\left(\left\|p_{k h}\left(\bar{y}_{\alpha}-y_{d}\right)-\bar{p}_{\alpha}\right\|_{L}+\left\|p_{k h}\left(y_{k h}\left(\bar{u}_{\alpha}\right)-\bar{y}_{\alpha}\right)\right\|_{L}\right)\left\|\bar{u}_{\alpha}-\bar{u}_{d}\right\|_{L^{1}\left(\Omega_{U}\right)} \\
\leq & C\left(\left\|p_{k h}\left(\bar{y}_{\alpha}-y_{d}\right)-\bar{p}_{\alpha}\right\|_{L}+\left\|p_{k h}\left(y_{k h}\left(\bar{u}_{\alpha}\right)-\bar{y}_{\alpha}\right)\right\|_{L}\right) . \\
& \quad\left(\alpha^{\kappa}+\left\|\left(p_{k h}-p\right)\left(y\left(\bar{u}_{d}\right)-y_{d}\right)\right\|_{L}^{\kappa}+\left\|p_{k h}\left(y_{k h}\left(\bar{u}_{d}\right)-y\left(\bar{u}_{d}\right)\right)\right\|_{L}^{\kappa}\right) .
\end{aligned}
$$

Invoking again Lemma 3.4, Lemma 3.1, estimate (3.9) from Lemma 3.2, and Lemma 2.6, we get

$$
\left\|\bar{y}_{d}-y_{k h}\left(\bar{u}_{\alpha}\right)\right\|_{I}^{2} \leq C\left(h^{2}+k\right) d_{0}\left(\bar{u}_{\alpha}\right)\left(\alpha^{\kappa}+\left(h^{2}+k\right)^{\kappa} d_{0}^{\kappa}\left(\bar{u}_{d}\right)\right),
$$

which is the auxiliary estimate (3.23) of the statement.

If $\kappa>1$, we can use the Cauchy-Schwarz inequality to get from it the estimate

$$
\left\|\bar{y}_{d}-y_{k h}\left(\bar{u}_{\alpha}\right)\right\|_{I}^{2} \leq C\left(\left(h^{2}+k\right)^{2} d_{0}^{2}\left(\bar{u}_{\alpha}\right)+\alpha^{2 \kappa}+\left(h^{2}+k\right)^{1+\kappa} d_{0}\left(\bar{u}_{\alpha}\right) d_{0}^{\kappa}\left(\bar{u}_{d}\right)\right) .
$$


Since $\kappa>1$, collecting all estimates yields the inequality

$$
\left\|\bar{y}_{d}-\bar{y}_{0}\right\|_{I}^{2} \leq C\left(\alpha^{2 \kappa}+\left(h^{2}+k\right)^{2} \max \left(d_{0}^{2 \kappa}\left(\bar{u}_{d}\right), d_{0}^{2}\left(\bar{u}_{\alpha}\right)\right)\right)
$$

from which we finally get (3.25).

Corollary 3.3. Let the assumptions of the previous theorem hold. For the adjoint state we have the error estimate

$$
\left\|\bar{p}_{0}-\bar{p}_{d}\right\|_{L^{\infty}\left(I, L^{2}(\Omega)\right)} \leq C\left(\alpha^{\max \left(\frac{1+\kappa}{2}, \kappa\right)}+\left(k+h^{2}\right)^{\min \left(1, \frac{1+\kappa}{2}\right)} C\left(\bar{u}_{d}, \bar{u}_{\alpha}\right)\right)
$$

with $C\left(\bar{u}_{d}, \bar{u}_{\alpha}\right)=\max \left(1, d_{0}\left(\bar{u}_{d}\right), d_{0}\left(\bar{u}_{\alpha}\right)\right)^{\max \left(1, \frac{1+\kappa}{2}\right)}$.

Proof. Inspecting the proof of Corollary 3.2, we get the estimate

$$
\left\|\bar{p}_{\alpha}-\bar{p}_{d}\right\|_{L^{\infty}\left(I, L^{2}(\Omega)\right)} \leq C\left(\left(k+h^{2}\right) d_{0}\left(\bar{u}_{\alpha}\right)+\left\|y_{k h}\left(\bar{u}_{\alpha}\right)-\bar{y}_{d}\right\|_{I}\right) .
$$

The last addend can be estimated with the auxiliary estimate (3.23) from the previous theorem and Cauchy's inequality. We obtain

$$
\left\|\bar{p}_{\alpha}-\bar{p}_{d}\right\|_{L^{\infty}\left(I, L^{2}(\Omega)\right)} \leq C\left(\alpha^{\max \left(\frac{1+\kappa}{2}, \kappa\right)}+\left(k+h^{2}\right)^{\min \left(1, \frac{1+\kappa}{2}\right)} C\left(\bar{u}_{d}, \bar{u}_{\alpha}\right)\right) .
$$

Invoking the regularization errors (2.20) and (2.22) proves the claim.

\subsection{Improved estimates for bang-bang controls}

As motivated at the end of subsection 3.2, improving the decay estimate (3.14) with the help of Lemma 2.8 leads to improved (non-robust) error estimates. However, the convergence rate $h^{2}$ is not achievable in these estimates, but the robust estimates from Theorem 3.2 overcome this problem. On the other hand, in Theorem 3.2 we have $\bar{u}_{d}$ on the right-hand side instead of $\bar{u}_{\alpha}$, so that Lemma 2.8 can not be directly applied. Therefor, we have to estimate some additional terms in combination with Theorem 3.2 to finally get the desired improved estimates.

Theorem 3.4. Let the assumptions of Theorem 3.2 be fulfilled. Further, we assume located controls and $A=\Omega_{U}$ up to a set of measure zero (measure condition on the whole domain). If $\kappa<1$, we additionally require the $\bar{p}_{\alpha}$-measure condition (2.29). (For $\kappa \geq 1$, this condition is automatically met as shown in $\left[12\right.$, Lemma 26].) Then, for $\alpha>0$ sufficiently small, $d_{0}:=d_{0}\left(\bar{u}_{\alpha}\right)$ given as in Theorem 3.1, and $C_{a b}$ defined in Lemma 2.8 it holds

$$
\left\|\bar{u}_{0}-\bar{u}_{d}\right\|_{U}^{2}+\left\|\bar{u}_{0}-\bar{u}_{d}\right\|_{L^{1}\left(\Omega_{U}\right)} \leq C\left(\alpha+h^{2}+k^{2} \max \left(1, C_{a b}, \alpha^{\kappa / 2-1}\right)\right)^{\kappa}\left(1+d_{0}^{\kappa}\right)
$$

for the error in the control.

Proof. Let us recall the estimate (3.20) from the proof of Theorem 3.2, i.e.,

$$
\begin{aligned}
& \left\|\bar{u}_{0}-\bar{u}_{d}\right\|_{L^{1}(A)}^{1+1 / \kappa}+\alpha\left\|\bar{u}_{0}-\bar{u}_{d}\right\|_{U}^{2}+\left\|y\left(\bar{u}_{0}\right)-y\left(\bar{u}_{d}\right)\right\|_{I}^{2} \\
\leq & C\left(-B^{*} p\left(y\left(\bar{u}_{d}\right)-y_{d}\right)+B^{*} p_{k h}\left(y_{k h}\left(\bar{u}_{d}\right)-y_{d}\right)+\alpha \bar{u}_{0}, \bar{u}_{0}-\bar{u}_{d}\right)_{U}
\end{aligned}
$$


which we rearrange as follows:

$$
\begin{aligned}
& \left\|\bar{u}_{0}-\bar{u}_{d}\right\|_{L^{1}(A)}^{1+1 / \kappa}+\alpha\left\|\bar{u}_{0}-\bar{u}_{d}\right\|_{U}^{2}+\left\|y_{k h}\left(\bar{u}_{0}\right)-y_{k h}\left(\bar{u}_{d}\right)\right\|_{I}^{2} \\
& \leq C(\underbrace{-B^{*} p\left(y\left(\bar{u}_{0}\right)-y\left(\bar{u}_{\alpha}\right)\right)}_{I} \underbrace{-B^{*} p\left(y\left(\bar{u}_{\alpha}\right)-y_{d}\right)+B^{*} p_{k h}\left(y_{k h}\left(\bar{u}_{\alpha}\right)-y_{d}\right)}_{I I a} \\
& \underbrace{+\alpha \bar{u}_{0}}_{I I b} \underbrace{+B^{*} p_{k h}\left(y_{k h}\left(\bar{u}_{0}\right)-y_{k h}\left(\bar{u}_{\alpha}\right)\right)}_{I I I}, \bar{u}_{0}-\bar{u}_{d})_{U} .
\end{aligned}
$$

For term $I I I$, we use the optimality conditions together with Cauchy's inequality to get

$$
\begin{aligned}
& \left.\left(y_{k h}\left(\bar{u}_{0}\right)-y_{k h}\left(\bar{u}_{\alpha}\right)\right), y_{k h}\left(\bar{u}_{0}\right)-y_{k h}\left(\bar{u}_{d}\right)\right)_{I} \\
\leq & C\left\|y_{k h}\left(\bar{u}_{0}\right)-y_{k h}\left(\bar{u}_{\alpha}\right)\right\|_{I}^{2}+\frac{1}{16}\left\|y_{k h}\left(\bar{u}_{0}\right)-y_{k h}\left(\bar{u}_{d}\right)\right\|_{I}^{2},
\end{aligned}
$$

and move the latter addend to the left-hand side of (3.28). We split the former addend with the help of (3.9) from Lemma 3.2 and the regularization errors (2.19) and (2.20) to obtain with the help of Young's inequality

$$
\begin{aligned}
& \left\|y_{k h}\left(\bar{u}_{0}\right)-y_{k h}\left(\bar{u}_{\alpha}\right)\right\|_{I}^{2} \leq C\left(\left\|\left(\hat{y}_{k h}-\hat{y}\right)\left(\bar{u}_{0}-\bar{u}_{\alpha}\right)\right\|_{I}+\left\|y\left(\bar{u}_{0}\right)-y\left(\bar{u}_{\alpha}\right)\right\|_{I}\right)^{2} \\
\leq & C\left(\left(k+h^{2}\right) \alpha^{\kappa / 2}+\alpha^{\frac{1+\kappa}{2}}\right)^{2} \leq C\left(k+h^{2}\right)^{2(\kappa+1)}+C \alpha^{1+\kappa},
\end{aligned}
$$

where $\hat{y}_{k h}$ and $\hat{y}$ denote the solution operators for the state equation with initial value zero.

For $I I b$, we invoke again Young's inequality and the inclusion $\bar{u}_{0} \in U_{\text {ad }} \subset L^{\infty}$ to get the estimate

$$
\alpha\left(\bar{u}_{0}, \bar{u}_{0}-\bar{u}_{d}\right)_{U} \leq C \alpha\left\|\bar{u}_{d}-\bar{u}_{0}\right\|_{L^{1}\left(\Omega_{U}\right)} \leq C \alpha^{\kappa+1}+\frac{1}{16}\left\|\bar{u}_{d}-\bar{u}_{0}\right\|_{L^{1}\left(\Omega_{U}\right)}^{1+1 / \kappa} .
$$

We now move the second summand to the left of (3.28) since $A=\Omega_{U}$ up to a set of measure zero. The addend $I I a$ can be rewritten and estimated with again the help of Young's inequality to get

$$
\begin{aligned}
& \left(-B^{*} p\left(y\left(\bar{u}_{\alpha}\right)-y_{d}\right)+B^{*} p_{k h}\left(y_{k h}\left(\bar{u}_{\alpha}\right)-y_{d}\right), \bar{u}_{0}-\bar{u}_{d}\right)_{U} \\
\leq & C\left(B^{*}\left(p_{k h}-p\right)\left(y\left(\bar{u}_{\alpha}\right)-y_{d}\right)+B^{*} p_{k h}\left(y_{k h}\left(\bar{u}_{\alpha}\right)-y\left(\bar{u}_{\alpha}\right)\right), \bar{u}_{0}-\bar{u}_{d}\right)_{U} \\
\leq & C\left\|B^{*}\left(p_{k h}-p\right)\left(y\left(\bar{u}_{\alpha}\right)-y_{d}\right)+B^{*} p_{k h}\left(y_{k h}\left(\bar{u}_{\alpha}\right)-y\left(\bar{u}_{\alpha}\right)\right)\right\|_{L^{\infty}\left(\Omega_{U}\right)}^{1+\kappa}+\frac{1}{16}\left\|\bar{u}_{0}-\bar{u}_{d}\right\|_{L^{1}\left(\Omega_{U}\right)}^{1+1 / \kappa}
\end{aligned}
$$

The last addend can now be moved to the left of (3.28). For summand $I$, we add an additional term to get

$$
\begin{aligned}
& \left(-B^{*} p\left(y\left(\bar{u}_{0}\right)-y\left(\bar{u}_{\alpha}\right)\right), \bar{u}_{0}-\bar{u}_{d}\right)_{U} \\
= & \left(B^{*}\left(p_{k h}-p\right)\left(y\left(\bar{u}_{0}\right)-y\left(\bar{u}_{\alpha}\right)\right)-B^{*} p_{k h}\left(y\left(\bar{u}_{0}\right)-y\left(\bar{u}_{\alpha}\right)\right), \bar{u}_{0}-\bar{u}_{d}\right)_{U} .
\end{aligned}
$$

We estimate the second addend with the help of the regularization error (2.20) as

$$
\left(y\left(\bar{u}_{0}\right)-y\left(\bar{u}_{\alpha}\right), y_{k h}\left(\bar{u}_{0}\right)-y_{k h}\left(\bar{u}_{d}\right)\right)_{I} \leq C \alpha^{1+\kappa}+\frac{1}{16}\left\|y_{k h}\left(\bar{u}_{0}\right)-y_{k h}\left(\bar{u}_{d}\right)\right\|_{I}^{2},
$$


and move the second addend to the left of (3.28). For the remaining addend, we use again the above mentioned results and the estimate (3.26) to obtain

$$
\begin{aligned}
& \left(B^{*}\left(p_{k h}-p\right)\left(y\left(\bar{u}_{0}\right)-y\left(\bar{u}_{\alpha}\right)\right), \bar{u}_{0}-\bar{u}_{d}\right)_{U} \\
= & \left(y\left(\bar{u}_{0}\right)-y\left(\bar{u}_{\alpha}\right),\left(\hat{y}_{k h}-\hat{y}\right)\left(\bar{u}_{0}-\bar{u}_{d}\right)\right) \\
\leq & C\left\|y\left(\bar{u}_{0}\right)-y\left(\bar{u}_{\alpha}\right)\right\|_{I}^{2}+C\left\|\left(\hat{y}_{k h}-\hat{y}\right)\left(\bar{u}_{0}-\bar{u}_{d}\right)\right\|_{I}^{2} \\
\leq & C \alpha^{1+\kappa}+C\left(k+h^{2}\right)^{2}\left\|\bar{u}_{0}-\bar{u}_{d}\right\|_{U}^{2} \\
\leq & C \alpha^{1+\kappa}+C\left(k+h^{2}\right)^{2}\left\|\bar{u}_{0}-\bar{u}_{d}\right\|_{L^{1}\left(\Omega_{U}\right)} \\
\leq & C \alpha^{1+\kappa}+C\left(k+h^{2}\right)^{2(\kappa+1)}+\frac{1}{16}\left\|\bar{u}_{0}-\bar{u}_{d}\right\|_{L^{1}\left(\Omega_{U}\right)}^{1+1 / \kappa}
\end{aligned}
$$

and move the last term to the left of (3.28).

Collecting all previous estimates, we with $L:=L^{\infty}\left(I, L^{2}(\Omega)\right)$ obtain

$$
\begin{aligned}
& \left\|\bar{u}_{0}-\bar{u}_{d}\right\|_{L^{1}(A)}^{1+1 / \kappa}+\alpha\left\|\bar{u}_{0}-\bar{u}_{d}\right\|_{U}^{2}+\left\|y_{k h}\left(\bar{u}_{0}\right)-y_{k h}\left(\bar{u}_{d}\right)\right\|_{I}^{2} \\
& \leq C\left(\alpha^{\kappa+1}+\left(k+h^{2}\right)^{2(\kappa+1)}+\left\|\left(p_{k h}-p\right)\left(y\left(\bar{u}_{\alpha}\right)-y_{d}\right)\right\|_{L}^{1+\kappa}\right. \\
& \left.\quad+\left\|p_{k h}\left(y_{k h}\left(\bar{u}_{\alpha}\right)-\mathcal{P}_{Y_{k}} y\left(\bar{u}_{\alpha}\right)\right)\right\|_{L}^{1+\kappa}\right) .
\end{aligned}
$$

Note that we introduced the orthogonal projection $\mathcal{P}_{Y_{k}}$ in the last addend, which is possible due to the definition of the fully discrete adjoint equation (3.7). Furthermore, we used stability of $B^{*}$ for located controls.

We combine the previous estimate with the (improved) adjoint error estimate from Lemma 3.4, the adjoint stability from Lemma 3.1, and the superconvergence result from Lemma 3.5, making use of the regularity given in Lemma 2.6, to get

$$
\begin{aligned}
& \left\|\bar{u}_{0}-\bar{u}_{d}\right\|_{L^{1}(A)}^{1+1 / \kappa}+\alpha\left\|\bar{u}_{0}-\bar{u}_{d}\right\|_{U}^{2}+\left\|y_{k h}\left(\bar{u}_{0}\right)-y_{k h}\left(\bar{u}_{d}\right)\right\|_{I}^{2} \\
\leq & C\left(\alpha+h^{2} d_{0}+k^{2}\left(1+d_{1}^{+}\left(\bar{u}_{\alpha}\right)\right)\right)^{1+\kappa} .
\end{aligned}
$$

With the help of the estimate given in Lemma 2.8 for $p=2$, i.e.,

$$
\left\|\partial_{t} \bar{u}_{\alpha}\right\|_{L^{2}\left(\Omega_{U}\right)} \leq C \max \left(C_{a b}, \alpha^{\kappa / 2-1}\right),
$$

we conclude that for $\alpha>0$ sufficiently small it holds

$$
d_{1}^{+}\left(\bar{u}_{\alpha}\right) \leq C+C \max \left(C_{a b}, \alpha^{\kappa / 2-1}\right) .
$$

In conclusion, we get

$$
\begin{aligned}
& \left\|\bar{u}_{0}-\bar{u}_{d}\right\|_{L^{1}(A)}^{1+1 / \kappa}+\alpha\left\|\bar{u}_{0}-\bar{u}_{d}\right\|_{U}^{2}+\left\|y_{k h}\left(\bar{u}_{0}\right)-y_{k h}\left(\bar{u}_{d}\right)\right\|_{I}^{2} \\
\leq & C\left(\alpha+h^{2} d_{0}+k^{2} \max \left(1, C_{a b}, \alpha^{\kappa / 2-1}\right)\right)^{1+\kappa} .
\end{aligned}
$$

Finally, recall that the $U$ error in the control can be derived from the corresponding $L^{1}$ error using the estimate (3.26).

From the previous theorem we get coupling rules for $\alpha$ and $k$, always with $\alpha=h^{2}$, and convergence rates, which are shown in Table 3.1 .

Note that in any case we get a better rate than $k^{\kappa}$ proven in Theorem 3.3. 
Table 3.1: Coupling and convergence implied by Theorem 3.4.

\begin{tabular}{|c|c|c|}
\hline$\alpha=$ & $\left\|\bar{u}_{d}-\bar{u}_{0}\right\|_{L^{1}\left(\Omega_{U}\right)} \leq C \ldots$ & if \\
\hline$k^{4 /(4-\kappa)}$ & $\alpha^{\kappa}=h^{2 \kappa}=k^{4 \kappa /(4-\kappa)}$ & $\kappa<2$ \\
$k^{2}$ & $\alpha^{\kappa}=h^{2 \kappa}=k^{2 \kappa}$ & $\kappa \geq 2$ \\
\hline
\end{tabular}

Corollary 3.4. Let the assumptions of the previous Theorem hold. For the adjoint and the projected state we have the error estimate

$$
\begin{aligned}
& \left\|\bar{p}_{0}-\bar{p}_{d}\right\|_{L^{\infty}\left(I, L^{2}(\Omega)\right)}+\left\|\bar{y}_{0}-\pi_{P_{k}^{*}} \bar{y}_{d}\right\|_{I} \\
\leq & C \alpha^{\max \left(\frac{\kappa+1}{2}, \kappa\right)}+C\left(h^{2} d_{0}+k^{2} \max \left(1, C_{a b}, \alpha^{\kappa / 2-1}\right)\right)^{\min \left(1, \frac{\kappa+1}{2}\right)} .
\end{aligned}
$$

Proof. Inspecting the proof of Corollary 3.2, we obtain the estimate

$$
\left\|\bar{p}_{\alpha}-\bar{p}_{d}\right\|_{L^{\infty}\left(I, L^{2}(\Omega)\right)}+\left\|\bar{y}_{\alpha}-\pi_{P_{k}^{*}} \bar{y}_{d}\right\|_{I} \leq C\left(k^{2} d_{1}^{+}+h^{2} d_{0}+\left\|y_{k h}\left(\bar{u}_{\alpha}\right)-\bar{y}_{d}\right\|_{I}\right) .
$$

To estimate the last addend, let us first combine the estimate (3.30) from the proof of Theorem 3.4 with Remark 3.2 to get

$$
\left\|\bar{u}_{\alpha}-\bar{u}_{d}\right\|_{L^{1}(A)} \leq C\left(\alpha+h^{2} d_{0}+k^{2}\left(1+d_{1}^{+}\left(\bar{u}_{\alpha}\right)\right)\right)^{\kappa} .
$$

With this estimate, we now follow the proof of Theorem 3.3 from the entry point (3.27) onwards. We obtain

$$
\begin{aligned}
& \left\|\bar{p}_{\alpha}-\bar{p}_{d}\right\|_{L^{\infty}\left(I, L^{2}(\Omega)\right)}^{2}+\left\|\bar{y}_{\alpha}-\pi_{P_{k}^{*}} \bar{y}_{d}\right\|_{I}^{2} \\
\leq & C\left(\left(h^{2} d_{0}+k^{2} d_{1}^{+}\right)^{2}+\left(h^{2} d_{0}+k^{2} d_{1}^{+}\right)\left(\alpha+h^{2} d_{0}+k^{2}\left(1+d_{1}^{+}\right)\right)^{\kappa}\right) .
\end{aligned}
$$

With Young's inequality, the regularization error (2.20), property (2.21), and the decay estimate (3.31), we finally get the claim.

\section{Numerics}

We will now consider a test example in order to finally validate numerically the theoretical results.

As we have previously said, we solve numerically the regularized problem $\left(\mathbb{P}_{k h}\right)$ for some $\alpha>0$ as an approximation of the limit problem $\left(\mathbb{P}_{0}\right)$. Thus, we have the influence of two errors: The regularization error in dependence of the parameter $\alpha>0$ and the discretization error due to space and time approximation. The second error depends on the fineness of the space and time grid, respectively, thus on the parameters $h$ and $k$.

We do not investigate the time discretization error for fixed positive $h$ and $\alpha$ by taking $k \rightarrow 0$, since this can be found in [15]. The numerical behavior of the error if $h \rightarrow 0$, again for fixed $\alpha>0$ but now with fixed $k$ instead of $h$ is discussed in [12, section 3.1.2]. The regularization error for fixed small discretization parameters $k$ and $h$ in dependence of the parameter $\kappa$ from the measure condition (2.16) if $\alpha \rightarrow 0$ can be found in [17] or [12, section 3.2].

Here, we only report on the coupling of regularization and discretization parameters as proposed by Theorem 3.4 and Table 3.1. 
We make use of the fact that instead of the linear control operator $B$, given by (2.4), we can also use an affine linear control operator

$$
\tilde{B}: U \rightarrow L^{2}\left(I, H^{-1}(\Omega)\right), \quad u \mapsto g_{0}+B u
$$

where $g_{0}$ is a fixed function. If we assume that $g_{0}$ is an element of the space $H^{1}\left(I, L^{2}(\Omega)\right)$ with $g_{0}(0) \in H_{0}^{1}(\Omega)$ and $g_{0}(T) \in H_{0}^{1}(\Omega)$, the preceding theory remains valid since $g_{0}$ can be interpreted as a modification of $y_{d}$.

For the limit problem $\left(\mathbb{P}_{0}\right)$, we consider a test example which is a bang-bang problem with meas $\left(A^{c}\right)=0$ and $\kappa=1$ in Assumption 2.2.

With a space-time domain $\Omega \times I:=(0,1)^{2} \times(0,0.5)$, we consider a located control function $\bar{u}$ and a constant $a:=2$, not to be confused with the lower bound $a_{1}$ of the admissible set $U_{\text {ad }}$ defined below. This constant $a$ influences the number of switching points between the active and inactive set. Furthermore, we define the functions

$$
\begin{aligned}
& g_{1}\left(x_{1}, x_{2}\right):=\sin \left(\pi x_{1}\right) \sin \left(\pi x_{2}\right), \\
& w_{a}\left(t, x_{1}, x_{2}\right):=\cos \left(\frac{t}{T} 2 \pi a\right) \cdot g_{1}\left(x_{1}, x_{2}\right),
\end{aligned}
$$

and choose an optimal adjoint state

$$
\bar{p}:=\frac{-T}{2 \pi a} \sin \left(\frac{t}{T} 2 \pi a\right) g_{1}
$$

which is nonzero almost everywhere, and since

$$
-\partial_{t} \bar{p}-\Delta \bar{p}=\cos \left(\frac{t}{T} 2 \pi a\right) g_{1}-\frac{T}{2 \pi a} \sin \left(\frac{t}{T} 2 \pi a\right) 2 \pi^{2} g_{1}=\bar{y}-y_{d},
$$

we get the function $y_{d}$ by taking $\bar{y}$ as

$$
\bar{y}\left(t, x_{1}, x_{2}\right):=w_{a}\left(t, x_{1}, x_{2}\right) .
$$

From the relation (2.12) we conclude that the optimal control is given by

$$
\bar{u}= \begin{cases}a_{1} & \text { if } B^{*} \bar{p}>0 \\ b_{1} & \text { if } B^{*} \bar{p}<0\end{cases}
$$

Note that $B^{*} \bar{p}(t)=\left(g_{1}, \bar{p}(t)\right)_{L^{2}(\Omega)}$, the initial value of the optimal state $\bar{y}$ is

$$
y_{0}\left(x_{1}, x_{2}\right)=\bar{y}\left(0, x_{1}, x_{2}\right)=g_{1}\left(x_{1}, x_{2}\right),
$$

and $\left(g_{1}, g_{1}\right)_{L^{2}(\Omega)}=0.25$. We obtain

$$
g_{0}=g_{1} 2 \pi\left(-\frac{a}{T} \sin \left(\frac{t}{T} 2 \pi a\right)+\pi \cos \left(\frac{t}{T} 2 \pi a\right)\right)-B \bar{u}
$$

and finally define the bounds of the admissible set $U_{\text {ad }}$ as $a_{1}:=0.2$ and $b_{1}:=0.4$.

Since $\kappa=1$ in this example, we conclude with Theorem 3.4, Corollary 3.4, and the second line of Table 3.1 the estimate

$$
\begin{aligned}
& \left\|\bar{u}_{0}-\bar{u}_{d}\right\|_{U}^{2}+\left\|\bar{u}_{0}-\bar{u}_{d}\right\|_{L^{1}(A)}+\left\|\bar{p}_{0}-\bar{p}_{d}\right\|_{L^{\infty}\left(I, L^{2}(\Omega)\right)}+\left\|\bar{y}_{0}-\pi_{P_{k}^{*}} \bar{y}_{d}\right\|_{I} \\
\leq & C\left(\alpha+h^{2}+k^{4 / 3}\right) .
\end{aligned}
$$


Table 4.1: Errors and $h$-EOC in the control $\left(\alpha=k^{4 / 3}=h^{2}\right)$.

\begin{tabular}{|ccccc|}
\hline & $\left\|\bar{u}-u_{k h}\right\|$ & $\left\|\bar{u}-u_{k h}\right\|$ & EOC & EOC \\
$\ell$ & $L^{1}(I, \mathbb{R})$ & $L^{2}(I, \mathbb{R})$ & $L^{1}$ & $L^{2}$ \\
\hline 1 & 0.05208333 & 0.10206207 & $/$ & $/$ \\
2 & 0.05156250 & 0.10155048 & 0.01 & 0.01 \\
3 & 0.01551730 & 0.05249039 & 1.73 & 0.95 \\
4 & 0.00395214 & 0.02696386 & 1.97 & 0.96 \\
5 & 0.00100074 & 0.01375946 & 1.98 & 0.97 \\
6 & 0.00026290 & 0.00704586 & 1.93 & 0.97 \\
\hline
\end{tabular}

Table 4.2: Errors and $h$-EOC in the state $\left(\alpha=k^{4 / 3}=h^{2}\right)$.

\begin{tabular}{|ccccccc|}
\hline & $\left\|\bar{y}-y_{k h}\right\|$ & $\left\|\bar{y}-y_{k h}\right\|$ & $\left\|\bar{y}-y_{k h}\right\|$ & EOC & EOC & EOC \\
$\ell$ & $L^{1}\left(I, L^{1}(\Omega)\right)$ & $L^{2}\left(I, L^{2}(\Omega)\right)$ & $L^{\infty}\left(I, L^{\infty}(\Omega)\right)$ & $L^{1}$ & $L^{2}$ & $L^{\infty}$ \\
\hline 1 & 0.04168338 & 0.14344433 & 0.77006182 & $/$ & $/$ & $/$ \\
2 & 0.02298795 & 0.05061771 & 0.24946457 & 0.86 & 1.50 & 1.63 \\
3 & 0.00877452 & 0.01795226 & 0.08863801 & 1.39 & 1.50 & 1.49 \\
4 & 0.00314952 & 0.00624197 & 0.02943581 & 1.48 & 1.52 & 1.59 \\
5 & 0.00111871 & 0.00218973 & 0.00994956 & 1.49 & 1.51 & 1.56 \\
6 & 0.00039580 & 0.00077075 & 0.00339060 & 1.50 & 1.51 & 1.55 \\
\hline
\end{tabular}

Consequently, we set $N h=\left(2^{\ell}+1\right)^{2}, \mathrm{Nk}=\left(2^{3 / 2 \ell+1}+1\right)$, and $\alpha=2^{-2 \ell}$ with $\ell=1,2,3,4,5,6$, to obtain second order convergence with respect to $h$ in (4.4).

We solve $\left(\mathbb{P}_{k h}\right)$ numerically with the above data using a fixed-point iteration for equation (3.11). Each fixed-point iteration is initialized with the starting value $u_{k h}^{(0)}:=a_{1}$ which is the lower bound of the admissible set. As a stopping criterion for the fixed-point iteration, we require for the discrete adjoint states belonging to the current and the last iterate that

$$
\left\|B^{*}\left(p_{k h}^{(i)}-p_{k h}^{(i-1)}\right)\right\|_{L^{\infty}(\Omega \times I)}<t_{0}
$$

where $t_{0}:=10^{-5}$ is a prescribed threshold.

The results are given in Tables 4.1-4.4. We also refer to Fig. 4.1.

As one can see from the tables, the coupling shows the expected behavior for the error in the optimal control, projected state, and adjoint state.

Note that for the state $\bar{y}$, we observe convergence of order $3 / 2$, which means by the coupling from above $\left(k=h^{3 / 2}\right)$ first order convergence in $k$. Thus, it is in accordance with our expectation since the state is discretized piecewise constant in time. This is depicted in Table 4.2.

A better and second order convergent approximation of the state is given by the projection $\pi_{P_{k}^{*}} y_{k h}$ of the computed discrete state $y_{k h}$, see Corollary 3.2 and for the corresponding numerical results see Table 4.3. This better approximation of the state can be obtained without further numerical effort: One only has to interpret the vector containing the values of $y_{k}$ on each interval $I_{m}$ as a vector of linearly-in-time linked values on the gridpoints of the dual grid $t_{1}^{*}<\cdots<t_{M}^{*}$.

Fig. 4.1 illustrates the convergence of $u_{k h}$ to $\bar{u}$. Note that the intersection points between the inactive set $\mathcal{I}_{k h}:=\left\{t \in I \mid a<u_{k h}(t)<b\right\}$ and the active set $\mathcal{A}_{k h}:=I \backslash \mathcal{I}_{k h}$ need not coincide with the time grid points since we use variational discretization for the control.

Let us mention that the convergence of the fixed-point iteration is in general guaranteed only for values of $\alpha$ not too small. This is an immediate consequence of Banach's fixed-point theorem 
Table 4.3: Errors and $h$-EOC in the projected state $\left(\alpha=k^{4 / 3}=h^{2}\right)$.

\begin{tabular}{|ccccccc|}
\hline & $\left\|\bar{y}-\pi_{P_{k}^{*}} y_{k h}\right\|$ & $\left\|\bar{y}-\pi_{P_{k}^{*}} y_{k h}\right\|$ & $\left\|\bar{y}-\pi_{P_{k}^{*}} y_{k h}\right\|$ & EOC & EOC & EOC \\
$\ell$ & $L^{1}\left(I, L^{1}(\Omega)\right)$ & $L^{2}\left(I, L^{2}(\Omega)\right)$ & $L^{\infty}\left(I, L^{\infty}(\Omega)\right)$ & $L^{1}$ & $L^{2}$ & $L^{\infty}$ \\
\hline 1 & 0.03984472 & 0.12699052 & 0.67616861 & $/$ & $/$ & $/$ \\
2 & 0.01063414 & 0.02423705 & 0.15855276 & 1.91 & 2.39 & 2.09 \\
3 & 0.00235558 & 0.00482756 & 0.02588151 & 2.17 & 2.33 & 2.61 \\
4 & 0.00059757 & 0.00116777 & 0.00526572 & 1.98 & 2.05 & 2.30 \\
5 & 0.00015345 & 0.00029551 & 0.00128779 & 1.96 & 1.98 & 2.03 \\
6 & 0.00003968 & 0.00007581 & 0.00032323 & 1.95 & 1.96 & 1.99 \\
\hline
\end{tabular}

Table 4.4: Errors and $h$-EOC in the adjoint state $\left(\alpha=k^{4 / 3}=h^{2}\right)$.

\begin{tabular}{|ccccccc|}
\hline & $\left\|\bar{p}-p_{k h}\right\|$ & $\left\|\bar{p}-p_{k h}\right\|$ & $\left\|\bar{p}-p_{k h}\right\|$ & EOC & EOC & EOC \\
$\ell$ & $L^{1}\left(I, L^{1}(\Omega)\right)$ & $L^{2}\left(I, L^{2}(\Omega)\right)$ & $L^{\infty}\left(I, L^{\infty}(\Omega)\right)$ & $L^{1}$ & $L^{2}$ & $L^{\infty}$ \\
\hline 1 & 0.00175355 & 0.00559389 & 0.02497779 & $/$ & $/$ & $/$ \\
2 & 0.00052886 & 0.00120225 & 0.00578048 & 1.73 & 2.22 & 2.11 \\
3 & 0.00012807 & 0.00026289 & 0.00128201 & 2.05 & 2.19 & 2.17 \\
4 & 0.00003156 & 0.00006214 & 0.00028508 & 2.02 & 2.08 & 2.17 \\
5 & 0.00000786 & 0.00001530 & 0.00006829 & 2.01 & 2.02 & 2.06 \\
6 & 0.00000195 & 0.00000377 & 0.00001649 & 2.01 & 2.02 & 2.05 \\
\hline
\end{tabular}

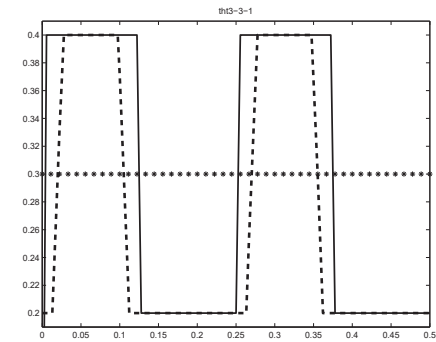

(a) $\ell=3$

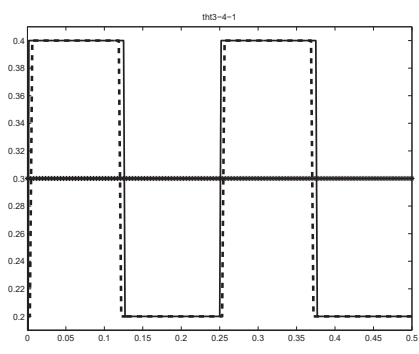

(b) $\ell=4$

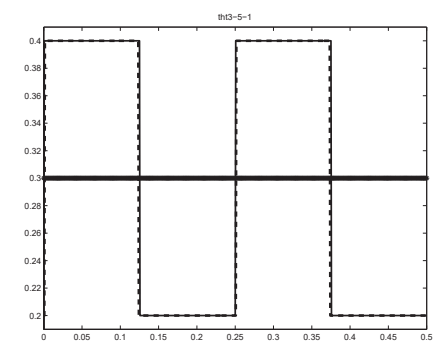

(c) $\ell=5$

Fig. 4.1. Optimal control $\bar{u}$ (solid) and computed counterpart $u_{k h}$ (dashed) over time after level $\ell$ $\left(\alpha=k^{4 / 3}=h^{2}\right)$.

in combination with (3.11). In the numerical examples we considered, no convergence problems occurred, even for very small values of $\alpha$. This might be due to the fact that we consider controls which "live" in one space dimension only. For higher dimensions, the situation is more delicate. There, the application of semismooth Newton methods has turned out to be fruitful, see [21] for its numerical analysis in the case of variational discretization of elliptic optimal control problems.

\section{References}

[1] K. Deckelnick and M. Hinze, A note on the approximation of elliptic control problems with bang-bang controls, Comput. Optim. Appl., 51 (2012), 931-939.

[2] D. Wachsmuth and G. Wachsmuth. Convergence and regularization results for optimal control problems with sparsity functional. In: ESAIM Control Optim. Calc. Var. 17:3 (2011), 858-886. 
[3] D. Wachsmuth and G. Wachsmuth, Regularization error estimates and discrepancy principle for optimal control problems with inequality constraints, Control Cybern., 40:4 (2011), 1125-1158.

[4] D. Wachsmuth and G. Wachsmuth, Necessary conditions for convergence rates of regularizations of optimal control problems, System Modelling and Optimization, Springer, (2013), 145-154.

[5] D. Wachsmuth, Adaptive regularization and discretization of bang-bang optimal control problems, ETNA, 40 (2013), 249-267.

[6] D. Wachsmuth, Robust error estimates for regularization and discretization of bang-bang control problems, Comput. Optim. Appl., 62 (2014), 271-289.

[7] W. Gong and N. Yan, Robust error estimates for the finite element approximation of elliptic optimal control problems, J. Comput. Appl. Math., 236:6 (2011), 1370-1381.

[8] U. Felgenhauer, On stability of bang-bang type controls, SIAM J. Control Optim., 41:6 (2003), $1843-1867$.

[9] W. Alt, R. Baier, M. Gerdts and F. Lempio, Error bounds for Euler approximation of linear-quadratic control problems with bang-bang solutions, NACO, 2:3 (2012), 547-570.

[10] W. Alt and M. Seydenschwanz, Regularization and discretization of linear-quadratic control problems, Control Cybern., 40:4 (2011), 903-920.

[11] M. Seydenschwanz, Convergence results for the discrete regularization of linear-quadratic control problems with bang-bang solutions, Comput. Optim. Appl., 61:3 (2015), 731-760.

[12] N. von Daniels, Bang-bang control of parabolic equations, Dissertation, Univ. Hamburg, 2016.

[13] M. Hinze, A variational discretization concept in control constrained optimization: The linearquadratic case, Computational Optimization and Applications, 30:1 (2005), 45-61.

[14] M. Hinze, R. Pinnau, M. Ulbrich and S. Ulbrich, Optimization with PDE Constraints, Springer, 2009.

[15] N. von Daniels, M. Hinze and M. Vierling, Crank-Nicolson time stepping and variational discretization of control-constrained parabolic optimal control problems, SIAM J. Control Optim., 53:3 (2015), 1182-1198.

[16] A. Springer and B. Vexler, Third order convergent time discretization for parabolic optimal control problems with control constraints, Comput. Optim. Appl., (2013), 1-36.

[17] N. von Daniels, Tikhonov regularization of control-constrained optimal control problems, Comput. Optim. Appl., 70:1 (2018), 295-320.

[18] L.C. Evans, Partial Differential Equations, AMS, 1998.

[19] S.C. Brenner and L.R. Scott, The Mathematical Theory of Finite Element Methods, Springer, 3rd ed., 2008.

[20] R.A. Adams and J.J. Fournier, Sobolev spaces, Elsevier Science Ltd, 2nd edition, 2003.

[21] M. Hinze and M. Vierling, The semi-smooth newton method for variationally discretized control constrained elliptic optimal control problems; implementation, convergence and globalization, Optim. Method Softw., 27:6 (2012), 933-950. 